\title{
3. UNDERWAY GEOPHYSICS: ODP LEG 103 ${ }^{1}$
}

\author{
Shipboard Scientific Party ${ }^{2}$
}

\section{INTRODUCTION}

Geophysical data were collected during Leg 103 of the Ocean Drilling Program (ODP). Of the $1741 \mathrm{n}$. mi traveled between Ponta Delgada, Azores, and Bremerhaven, Federal Republic of Germany, geophysical data were collected for approximately 250 $\mathrm{n}$. $\mathrm{mi}(28 \mathrm{hr})$ before arrival at Site 637 , when under way between the sites, and for approximately $30 \mathrm{n}$. $\mathrm{mi}(3 \mathrm{hr})$ after we departed final Site 641 . We were under way 8 days during the 54.8 days spent at sea.

Shipboard geophysical instrumentation included two precision echo-sounders, a magnetometer, seismic-reflection profilers, and a satellite navigation system. The instruments were maintained and operated by ODP marine technicians, in cooperation with the scientific party and the officers and crew of SEDCOFOREX, Inc.

\section{NAVIGATION DATA}

Navigation data were collected on the bridge by a Magnavox MX702A. Positions were obtained with this system during the

\footnotetext{
${ }^{1}$ Boillot, G., Winterer, E. L., Meyer, A. W., et al., Proc. Init. Repts. (Pt. A), $O D P, 103$.

${ }^{2}$ Gilbert Boillot (Co-Chief Scientist), Laboratoire de Géodynamique SousMarine, Université Pierre et Marie Curie, B.P. 48, 06230, Villefranche-sur-Mer, France; Edward L. Winterer (Co-Chief Scientist), Graduate Research Division A-012-W, Scripps Institution of Oceanography, La Jolla, CA 92093; Audrey W. Meyer (ODP Staff Scientist), Ocean Drilling Program, Texas A\&M University, College Station, TX 77843-3469; Joseph Applegate, Department of Geology, Florida State University, Tallahassee, FL 32306; Miriam Baltuck, Department of Geology, Tulane University, New Orleans, LA 70118 (current address: NASA Headquarters, Code EEL, Washington, D.C. 20546); James A. Bergen, Department of Geology, Florida State University, Tallahassee, FL 32306; M. C. Comas, Departamento Estratigrafia y Paleontologia, Facultad de Ciencias, Universidad de Granada, 18001 Granada, Spain; Thomas A. Davies, Institute for Geophysics, University of Texas at Austin, 4920 North IH 35, Austin, TX 78751; Keith Dunham, Department of Atmospheric and Oceanic Sciences, University of Michigan, 2455 Hayward Avenue, Ann Arbor, MI 48109 (current address: P.O. Box 13, Pequat Lakes, MN 56478); Cynthia A. Evans, Department of Geology, Colgate University, Hamilton, NY 13346 (current address: Lamont-Doherty Geological Observatory, Palisades, NY 10964); Jacques Girardeau, Laboratoire de Materiaux Terrestres, I.P.G., 4 Place Jussieu, 75252 Paris Cedex 05, France; David Goldberg, Lamont-Doherty Geological Observatory, Palisades, NY 10964; Janet Haggerty, Department of Geology, University of Tulsa, $600 \mathrm{~S}$. College Avenue, Tulsa, OK 74104; Lubomir F. Jansa, Atlantic Geoscience Center, Bedford Institute of Oceanography, Dartmouth, Nova Scotia B2Y 4A2, Canada; Jeffrey A. Johnson, Department of Earth and Space Sciences, University of California, Los Angeles, CA 90024 (current address: 9449 Briar Forest Drive, No. 3544, Houston, TX 77063); Junzo Kasahara, Earthquake Research Institute, University of Tokyo, 1,1,1 Yayoi, Bunkyo, Tokyo 113, Japan (current address: Nippon Schlumberger K.K., 2-1 Fuchinobe, 2-Chome, Sagamihara-shi, Kanagawa-ken, 229, Japan); Jean-Paul Loreau, Laboratoire de Géologie, Museum National D'Histoire Naturelle, 43 Rue Buffon, 75005 Paris, France; Emilio Luna, Hispanoil, Pez Volador No. 2, 28007 Madrid, Spain; Michel Moullade, Laboratoire de Géologie et Micropaleontologie Marines, Université de Nice, Parc Valrose, 06034 Nice Cedex, France; James Ogg, Geological Research Division A-012, Scripps Institution of Oceanography, La Jolla, CA 92093 (current address: Dept. Earth and Atmospheric Sciences, Purdue University, W. Lafayette, IN 47907); Massimo Sarti, Istituto di Geologia, Universitá di Ferrara, Corso Ercole d'Este, 32, 44100 Ferrara, Italy; Jürgen Thurow, Institut und Museum für Geologie und Paleontologie, Universität Tuebingen, Sigwartstr. 10, D-7400 Tuebingen, Federal Republic of Germany; Mark A. Williamson, Atlantic Geoscience Centre, Geological Survey of Canada, Bedford Institute of Oceanography, Box 1006, Dartmouth, Nova Scotia B2Y 4A2, Canada (current address: Shell Canada Ltd., P.O. Box 100, Stn. M, Calgary, Alberta T2P 2H5, Canada).
}

entire 54.8 days spent at sea. Approximately nine satellite fixes were received each day. Latitudes and longitudes of the drill holes were determined by averaging all the satellite fixes received while drilling each hole. These locations may differ slightly from the locations of the drill holes given in site chapters, which are the average of only the 20 best fixes received.

A plot of the general navigation from Leg 103 is shown in Figure 1A; an enlarged plot of the navigation around Leg 103 drill sites is shown in Figure 1B. The plots were generated from satellite navigation and course- and speed-change information (Table 1). These data were compiled from the shipboard bridge log, underway geophysical $\log$, and satellite-navigation data sheets; course and speed information were from the digital seismic tape headers. The Geological Data Center at Scripps Institution of Oceanography produced this navigation compilation.

More detailed navigation plots around each drill site were compiled by the Co-Chief Scientists during the cruise. These plots for Sites 637, 638, 639, and 640 are shown in Figures 2, 3, 4 , and 5 , respectively.

\section{BATHYMETRIC DATA}

Bathymetric data were obtained with both $3.5-\mathrm{kHz}$ (Raytheon recorder system) and $12-\mathrm{kHz}$ (EDO $248 \mathrm{C}$ recorder system) echosounders. Unfortunately, because of poor transducer location, the quality of the recorded data was poor when the ship was traveling at speeds greater than $6 \mathrm{kt}$. Consequently, site surveys requiring detailed bathymetric data were conducted at speeds slower than $6 \mathrm{kt}$ (see site chapters, this volume). Pertinent segments of these bathymetric data are presented in the site chapters and are not included in this chapter.

\section{MAGNETIC DATA}

Because of technical and electronic problems, no magnetic data were collected during Leg 103.

\section{SEISMIC-REFLECTION DATA}

The seismic sources used aboard the JOIDES Resolution during Leg 103 were two 80 -in. ${ }^{3}$ water guns. One 100 -m-long Teledyne streamer, deployed from the fantail and containing 60 active sections, was towed approximately $500 \mathrm{~m}$ behind the vessel. Towing depth was set by external depth depressors (birds). The hydrophone elements were combined to produce a single signal.

Seismic data were displayed in real time in analog format on two EDO 550 dry-paper recorders, using only streamers, amplifier, and two band-pass filters (Table 2).

Seismic data were also recorded using a super-micro 561 Masscomp computer, which functions as the central unit to record, process, and display the data. Data were processed and displayed in real-time on a 15-in.-wide Printronix high-resolution graphic printer (160 dots/in.). Raw data were recorded on Cipher tapes, using a SEG-Y format and a density of 1600 bytes/in. Seismic lines recorded with the Masscomp were reprocessed while the ship was on station. The final data were displayed on a 22-in.wide Versatec plotter ( 200 dots/in.). Table 3 gives the reprocessing parameters. 

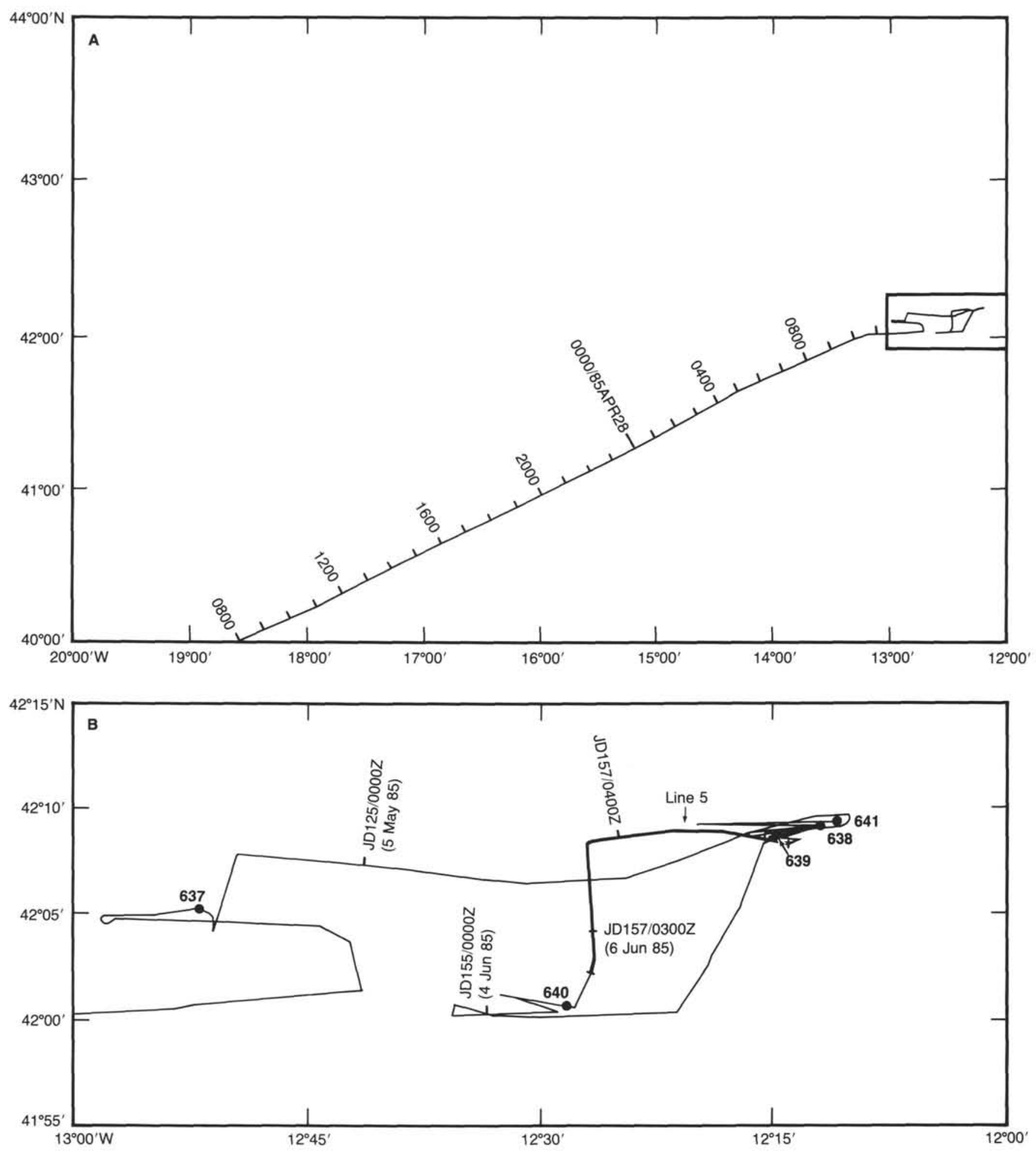

Figure 1. A General trackline map of ODP Leg 103 cruise, generated from satellite-navigation and course- and speed-change data given in Table 1. Area of enlarged map shown in Figure 1B is outlined. B Enlarged trackline map of Leg 103 cruise near drill sites. See Figure 1A for location. More detailed navigation plots around each drill site are shown in Figures 2 through 5 . Location of seismic line 5 (see Figs. 13 and 14) is shown by heavy line. 
Table 1. Satellite navigation and course- and speed-change data used to generate Leg 103 trackline plots shown in Figure 1.

\begin{tabular}{|c|c|c|c|c|c|c|c|c|c|c|c|c|c|c|c|}
\hline & & & & & rth & & & & & ual & & & reck & $\begin{array}{l}\text { ad } \\
\text { ning }\end{array}$ & \\
\hline & & & & & & & & & speed & course & speed & hed & speed & course & \\
\hline Day & Month & Year & Time $^{a}$ & deg & $\min$ & deg & $\min$ & Dist & $(\mathrm{kt})$ & (deg) & $(\mathrm{kt})$ & (deg) & (kt) & (deg) & Comments $^{\mathrm{b}}$ \\
\hline 27 & 4 & 1985 & 0512 & 39 & 48.50 & -19 & 9.60 & 0.0 & 10.9 & 66 & 2.5 & 73 & 8.5 & 64 & satl \\
\hline 27 & 4 & 1985 & 0700 & 39 & 56.46 & -18 & 46.17 & 19.7 & 9.9 & 62 & 1.5 & 54 & 8.5 & 64 & satl \\
\hline 27 & 4 & 1985 & 0824 & 40 & 2.88 & -18 & 30.09 & 33.6 & 11.3 & 67 & 2.9 & 75 & 8.5 & 64 & satl \\
\hline 27 & 4 & 1985 & 1108 & 40 & 15.11 & -17 & 52.99 & 64.4 & 11.1 & 62 & 2.6 & 57 & 8.5 & 64 & satl \\
\hline 27 & 4 & 1985 & 1256 & 40 & 24.36 & -17 & 29.76 & 84.4 & 10.7 & 65 & 2.2 & 68 & 8.5 & 64 & satl \\
\hline 27 & 4 & 1985 & 1720 & 40 & 44.4 & -16 & 33.8 & 131.5 & 10.7 & 65 & 2.2 & 68 & 8.5 & 64 & $\mathrm{c} / \mathrm{cs}$ \\
\hline 27 & 4 & 1985 & 1750 & 40 & 46.7 & -16 & 27.4 & 136.8 & 10.8 & 65 & 2.2 & 68 & 8.6 & 64 & $\mathrm{c} / \mathrm{cs}$ \\
\hline 27 & 4 & 1985 & 1814 & 40 & 48.57 & -16 & 22.21 & 141.1 & 11.1 & 65 & 2.5 & 66 & 8.6 & 64 & satl \\
\hline 27 & 4 & 1985 & 1816 & 40 & 48.7 & -16 & 21.8 & 141.5 & 11.3 & 65 & 2.5 & 66 & 8.8 & 64 & $\mathrm{c} / \mathrm{cs}$ \\
\hline 27 & 4 & 1985 & 1843 & 40 & 50.9 & -16 & 15.7 & 146.6 & 11.2 & 63 & 2.5 & 66 & 8.7 & 62 & $\mathrm{c} / \mathrm{cs}$ \\
\hline 27 & 4 & 1985 & 1905 & 40 & 52.8 & -16 & 10.9 & 150.7 & 11.2 & 62 & 2.5 & 66 & 8.7 & 61 & $\mathrm{c} / \mathrm{cs}$ \\
\hline 27 & 4 & 1985 & 1914 & 40 & 53.57 & -16 & 8.90 & 152.4 & 10.8 & 63 & 2.2 & 72 & 8.7 & 61 & satl \\
\hline 27 & 4 & 1985 & 1934 & 40 & 55.2 & -16 & 4.6 & 156.0 & 10.7 & 63 & 2.2 & 72 & 8.5 & 61 & $\mathrm{c} / \mathrm{cs}$ \\
\hline 27 & 4 & 1985 & 1959 & 40 & 57.2 & -15 & 59.4 & 160.4 & 10.6 & 62 & 2.2 & 72 & 8.5 & 60 & $\mathrm{c} / \mathrm{cs}$ \\
\hline 27 & 4 & 1985 & 2021 & 40 & 59.0 & -15 & 54.8 & 164.3 & 10.7 & 63 & 2.2 & 72 & 8.5 & 61 & $\mathrm{c} / \mathrm{cs}$ \\
\hline 27 & 4 & 1985 & 2038 & 41 & 0.4 & -15 & 51.2 & 167.4 & 10.5 & 63 & 2.2 & 72 & 8.4 & 61 & $\mathrm{c} / \mathrm{cs}$ \\
\hline 27 & 4 & 1985 & 2058 & 41 & 2.0 & -15 & 47.1 & 170.9 & 10.4 & 63 & 2.2 & 72 & 8.3 & 61 & $\mathrm{c} / \mathrm{cs}$ \\
\hline 27 & 4 & 1985 & 2120 & 41 & 3.7 & -15 & 42.5 & 174.7 & 10.1 & 63 & 2.2 & 72 & 8.0 & 61 & $\mathrm{c} / \mathrm{cs}$ \\
\hline 27 & 4 & 1985 & 2137 & 41 & 5.0 & -15 & 39.1 & 177.6 & 10.1 & 64 & 2.2 & 72 & 7.9 & 62 & $\mathrm{c} / \mathrm{cs}$ \\
\hline 27 & 4 & 1985 & 2158 & 41 & 6.5 & -15 & 34.9 & 181.1 & 10.5 & 64 & 2.2 & 72 & 8.3 & 62 & $\mathrm{c} / \mathrm{cs}$ \\
\hline 27 & 4 & 1985 & 2203 & 41 & 6.9 & -15 & 33.9 & 182.0 & 10.7 & 64 & 2.2 & 72 & 8.5 & 62 & $\mathrm{c} / \mathrm{cs}$ \\
\hline 27 & 4 & 1985 & 2219 & 41 & 8.1 & -15 & 30.5 & 184.8 & 10.0 & 63 & 2.2 & 72 & 7.9 & 61 & $\mathrm{c} / \mathrm{cs}$ \\
\hline 27 & 4 & 1985 & 2225 & 41 & 8.6 & -15 & 29.3 & 185.8 & 9.6 & 63 & 2.2 & 72 & 7.4 & 61 & $\mathrm{c} / \mathrm{cs}$ \\
\hline 27 & 4 & 1985 & 2242 & 41 & 9.8 & -15 & 26.1 & 188.5 & 9.7 & 64 & 2.2 & 72 & 7.6 & 62 & $\mathrm{c} / \mathrm{cs}$ \\
\hline 27 & 4 & 1985 & 2311 & 41 & 11.8 & -15 & 20.5 & 193.2 & 9.1 & 64 & 2.2 & 72 & 7.0 & 62 & $\mathrm{c} / \mathrm{cs}$ \\
\hline 27 & 4 & 1985 & 2322 & 41 & 12.6 & -15 & 18.4 & 194.9 & 9.1 & 64 & 2.2 & 72 & 6.9 & 62 & $\mathrm{c} / \mathrm{cs}$ \\
\hline 27 & 4 & 1985 & 2328 & 41 & 13.0 & -15 & 17.4 & 195.8 & 9.1 & 64 & 2.2 & 72 & 6.9 & 62 & $\mathrm{c} / \mathrm{cs}$ \\
\hline 27 & 4 & 1985 & 2349 & 41 & 14.3 & -15 & 13.5 & 199.0 & 9.4 & 64 & 2.2 & 72 & 7.3 & 62 & $\mathrm{c} / \mathrm{cs}$ \\
\hline 28 & 4 & 1985 & 0000 & 41 & 15.1 & -15 & 11.5 & 200.7 & 9.4 & 64 & 2.2 & 72 & 7.3 & 62 & $\mathrm{c} / \mathrm{cs}$ \\
\hline 28 & 4 & 1985 & 0004 & 41 & 15.4 & -15 & 10.7 & 201.4 & 8.9 & 64 & 2.2 & 72 & 6.8 & 62 & $\mathrm{c} / \mathrm{cs}$ \\
\hline 28 & 4 & 1985 & 0017 & 41 & 16.2 & -15 & 8.4 & 203.3 & 8.9 & 64 & 2.2 & 72 & 6.8 & 62 & $\mathrm{c} / \mathrm{cs}$ \\
\hline 28 & 4 & 1985 & 0020 & 41 & 16.40 & -15 & 7.86 & 203.7 & 9.2 & 59 & 2.4 & 51 & 6.8 & 62 & satl \\
\hline 28 & 4 & 1985 & 0032 & 41 & 17.3 & -15 & 5.8 & 205.6 & 9.9 & 59 & 2.4 & 51 & 7.5 & 62 & $\mathrm{c} / \mathrm{cs}$ \\
\hline 28 & 4 & 1985 & 0042 & 41 & 18.2 & -15 & 3.9 & 207.2 & 9.3 & 60 & 2.4 & 51 & 6.9 & 63 & $\mathrm{c} / \mathrm{cs}$ \\
\hline 28 & 4 & 1985 & 0210 & 41 & 25.06 & -14 & 48.10 & 220.9 & 9.2 & 63 & 2.2 & 64 & 6.9 & 63 & satl \\
\hline 28 & 4 & 1985 & 0212 & 41 & 25.2 & -14 & 47.7 & 221.2 & 9.4 & 61 & 2.2 & 64 & 7.2 & 60 & $\mathrm{c} / \mathrm{cs}$ \\
\hline 28 & 4 & 1985 & 0223 & 41 & 26.0 & -14 & 45.7 & 222.9 & 9.2 & 72 & 2.2 & 64 & 7.1 & 74 & $\mathrm{c} / \mathrm{cs}$ \\
\hline 28 & 4 & 1985 & 0229 & 41 & 26.3 & -14 & 44.6 & 223.9 & 9.5 & 65 & 2.2 & 64 & 7.3 & 65 & $\mathrm{c} / \mathrm{cs}$ \\
\hline 28 & 4 & 1985 & 0233 & 41 & 26.6 & -14 & 43.8 & 224.5 & 9.0 & 59 & 2.2 & 64 & 6.8 & 58 & $\mathrm{c} / \mathrm{cs}$ \\
\hline 28 & 4 & 1985 & 0239 & 41 & 27.1 & -14 & 42.8 & 225.4 & 8.9 & 60 & 2.2 & 64 & 6.7 & 58 & $\mathrm{c} / \mathrm{cs}$ \\
\hline 28 & 4 & 1985 & 0254 & 41 & 28.2 & -14 & 40.2 & 227.6 & 9.4 & 59 & 2.2 & 64 & 7.2 & 58 & $\mathrm{c} / \mathrm{cs}$ \\
\hline 28 & 4 & 1985 & 0314 & 41 & 29.8 & -14 & 36.6 & 230.8 & 9.3 & 59 & 2.2 & 64 & 7.1 & 58 & $\mathrm{c} / \mathrm{cs}$ \\
\hline 28 & 4 & 1985 & 0331 & 41 & 31.1 & -14 & 33.6 & 233.4 & 9.1 & 59 & 2.2 & 64 & 6.9 & 58 & $\mathrm{c} / \mathrm{cs}$ \\
\hline 28 & 4 & 1985 & 0334 & 41 & 32.1 & -14 & 31.3 & 235.4 & 9.2 & 59 & 2.2 & 64 & 7.0 & 58 & $\mathrm{c} / \mathrm{cs}$ \\
\hline 28 & 4 & 1985 & 0350 & 41 & 32.6 & -14 & 30.2 & 236.3 & 9.7 & 59 & 2.2 & 64 & 7.5 & 58 & $\mathrm{c} / \mathrm{cs}$ \\
\hline 28 & 4 & 1985 & 0355 & 41 & 33.0 & -14 & 29.3 & 237.1 & 9.2 & 59 & 2.2 & 64 & 7.0 & 58 & $\mathrm{c} / \mathrm{cs}$ \\
\hline 28 & 4 & 1985 & 0405 & 41 & 33.8 & -14 & 27.5 & 238.6 & 9.6 & 59 & 2.2 & 64 & 7.4 & 58 & $\mathrm{c} / \mathrm{cs}$ \\
\hline 28 & 4 & 1985 & 0423 & 41 & 35.2 & -14 & 24.2 & 241.5 & 9.6 & 60 & 2.2 & 64 & 7.4 & 59 & $\mathrm{c} / \mathrm{cs}$ \\
\hline 28 & 4 & 1985 & 0448 & 41 & 37.22 & -14 & 19.61 & 245.5 & 9.4 & 61 & 2.1 & 70 & 7.4 & 59 & satl \\
\hline 28 & 4 & 1985 & 0453 & 41 & 37.6 & -14 & 18.7 & 246.3 & 9.4 & 61 & 2.1 & 70 & 7.3 & 59 & $\mathrm{c} / \mathrm{cs}$ \\
\hline 28 & 4 & 1985 & 0510 & 41 & 38.9 & -14 & 15.6 & 248.9 & 9.5 & 65 & 2.1 & 70 & 7.4 & 64 & $\mathrm{c} / \mathrm{cs}$ \\
\hline 28 & 4 & 1985 & 0532 & 41 & 40.3 & -14 & 11.3 & 252.4 & 9.6 & 66 & 2.1 & 70 & 7.5 & 65 & $\mathrm{c} / \mathrm{cs}$ \\
\hline 28 & 4 & 1985 & 0542 & 41 & 41.0 & -14 & 9.3 & 254.0 & 9.4 & 66 & 2.1 & 70 & 7.3 & 65 & $\mathrm{c} / \mathrm{cs}$ \\
\hline 28 & 4 & 1985 & 0559 & 41 & 42.1 & -14 & 6.1 & 256.7 & 9.7 & 66 & 2.1 & 70 & 7.6 & 65 & $\mathrm{c} / \mathrm{cs}$ \\
\hline 28 & 4 & 1985 & 0611 & 41 & 42.8 & -14 & 3.7 & 258.6 & 9.5 & 66 & 2.1 & 70 & 7.3 & 65 & $\mathrm{c} / \mathrm{cs}$ \\
\hline 28 & 4 & 1985 & 0629 & 41 & 44.0 & -14 & 0.2 & 261.5 & 9.5 & 66 & 2.1 & 70 & 7.4 & 65 & $\mathrm{c} / \mathrm{cs}$ \\
\hline 28 & 4 & 1985 & 0636 & 41 & 44.4 & -13 & 58.9 & 262.6 & 9.3 & 66 & 2.1 & 70 & 7.2 & 65 & $\mathrm{c} / \mathrm{cs}$ \\
\hline 28 & 4 & 1985 & 0638 & 41 & 44.56 & -13 & 58.51 & 262.9 & 9.2 & 67 & 2.1 & 73 & 7.2 & 65 & satl \\
\hline 28 & 4 & 1985 & 0644 & 41 & 44.9 & -13 & 57.4 & 263.8 & 9.6 & 67 & 2.1 & 73 & 7.6 & 65 & $\mathrm{c} / \mathrm{cs}$ \\
\hline 28 & 4 & 1985 & 0702 & 41 & 46.1 & -13 & 53.8 & 266.7 & 9.5 & 67 & 2.1 & 73 & 7.5 & 65 & $\mathrm{c} / \mathrm{cs}$ \\
\hline 28 & 4 & 1985 & 0710 & 41 & 46.57 & -13 & 52.24 & 268.0 & 10.1 & 66 & 2.6 & 69 & 7.5 & 65 & satl \\
\hline 28 & 4 & 1985 & 0712 & 41 & 46.7 & -13 & 51.8 & 268.3 & 10.4 & 66 & 2.6 & 69 & 7.8 & 65 & $\mathrm{c} / \mathrm{cs}$ \\
\hline 28 & 4 & 1985 & 0724 & 41 & 47.5 & -13 & 49.3 & 270.4 & 10.0 & 66 & 2.6 & 69 & 7.4 & 65 & $\mathrm{c} / \mathrm{cs}$ \\
\hline 28 & 4 & 1985 & 0750 & 41 & 49.3 & -13 & 44.0 & 274.7 & 10.2 & 66 & 2.6 & 69 & 7.6 & 65 & $\mathrm{c} / \mathrm{cs}$ \\
\hline 28 & 4 & 1985 & 0814 & 41 & 50.9 & -13 & 39.0 & 278.8 & 10.6 & 66 & 2.6 & 69 & 8.0 & 65 & $\mathrm{c} / \mathrm{cs}$ \\
\hline 28 & 4 & 1985 & 0824 & 41 & 51.7 & -13 & 36.8 & 280.6 & 10.6 & 66 & 2.6 & 69 & 8.0 & 65 & $\mathrm{c} / \mathrm{cs}$ \\
\hline 28 & 4 & 1985 & 0847 & 41 & 53.3 & -13 & 31.8 & 284.6 & 10.5 & 67 & 2.6 & 69 & 7.9 & 66 & $\mathrm{c} / \mathrm{cs}$ \\
\hline 28 & 4 & 1985 & 0856 & 41 & 53.94 & -13 & 29.85 & 286.2 & 9.6 & 66 & 1.7 & 69 & 7.9 & 66 & satl \\
\hline 28 & 4 & 1985 & 0857 & 41 & 54.0 & -13 & 29.7 & 286.4 & 9.8 & 66 & 1.7 & 69 & 8.1 & 65 & $\mathrm{c} / \mathrm{cs}$ \\
\hline 28 & 4 & 1985 & 0911 & 41 & 54.9 & -13 & 26.9 & 288.6 & 9.4 & 66 & 1.7 & 69 & 7.8 & 65 & $\mathrm{c} / \mathrm{cs}$ \\
\hline 28 & 4 & 1985 & 0927 & 41 & 56.0 & -13 & 23.8 & 291.2 & 9.7 & 66 & 1.7 & 69 & 8.0 & 65 & $\mathrm{c} / \mathrm{cs}$ \\
\hline 28 & 4 & 1985 & 0943 & 41 & 57.0 & -13 & 20.6 & 293.7 & 9.9 & 66 & 1.7 & 69 & 8.2 & 65 & $\mathrm{c} / \mathrm{cs}$ \\
\hline 28 & 4 & 1985 & 1002 & 41 & 58.34 & -13 & 16.78 & 296.9 & 10.6 & 72 & 2.7 & 93 & 8.2 & 65 & satl \\
\hline 28 & 4 & 1985 & 1003 & 41 & 58.4 & -13 & 16.6 & 297.1 & 10.6 & 72 & 2.7 & 93 & 8.2 & 65 & $\mathrm{c} / \mathrm{cs}$ \\
\hline 28 & 4 & 1985 & 1026 & 41 & 59.7 & -13 & 11.4 & 301.1 & 10.4 & 72 & 2.7 & 93 & 8.0 & 65 & $\mathrm{c} / \mathrm{cs}$ \\
\hline 28 & 4 & 1985 & 1032 & 41 & 60.0 & -13 & 10.0 & 302.2 & 9.0 & 84 & 2.7 & 93 & 6.4 & 80 & $\mathrm{c} / \mathrm{cs}$ \\
\hline 28 & 4 & 1985 & 1034 & 42 & 0.0 & -13 & 9.6 & 302.5 & 7.0 & 88 & 2.7 & 93 & 4.3 & 84 & $\mathrm{c} / \mathrm{cs}$ \\
\hline 28 & 4 & 1985 & 1046 & 42 & 0.07 & -13 & 7.75 & 303.9 & 5.6 & 91 & 1.4 & 114 & 4.3 & 84 & satl \\
\hline 28 & 4 & 1985 & 1049 & 42 & 0.1 & -13 & 7.4 & 304.1 & 6.0 & 91 & 1.4 & 114 & 4.7 & 84 & $\mathrm{c} / \mathrm{cs}$ \\
\hline 28 & 4 & 1985 & 1107 & 42 & 0.0 & -13 & 4.9 & 305.9 & 6.0 & 88 & 1.4 & 114 & 4.8 & 81 & $\mathrm{c} / \mathrm{cs}$ \\
\hline
\end{tabular}


Table 1 (continued).

\begin{tabular}{|c|c|c|c|c|c|c|c|c|c|c|c|c|c|c|c|}
\hline & & & & & & & & & & & D & & $\begin{array}{r}\mathrm{D} \\
\text { reck }\end{array}$ & $\begin{array}{l}\text { ad } \\
\text { ning }\end{array}$ & \\
\hline Day & Month & Year & Time $^{\mathrm{a}}$ & deg & $\frac{\min }{}$ & $\frac{10 i n}{\operatorname{deg}}$ & min & Dist & $\begin{array}{c}\text { speed } \\
(\mathrm{kt})\end{array}$ & $\begin{array}{c}\text { course } \\
\text { (deg) }\end{array}$ & $\begin{array}{c}\text { speed } \\
(\mathrm{kt})\end{array}$ & $\begin{array}{l}\text { hed } \\
\text { (deg) }\end{array}$ & $\begin{array}{c}\text { speed } \\
(\mathrm{kt})\end{array}$ & $\begin{array}{c}\text { course } \\
\text { (deg) }\end{array}$ & Comments $^{b}$ \\
\hline 28 & 4 & 1985 & 1134 & 42 & 0.1 & -13 & 1.3 & 308.6 & 6.0 & 90 & 1.5 & 123 & 4.8 & 80 & $\mathrm{c} / \mathrm{cs}$ \\
\hline 28 & 4 & 1985 & 1154 & 42 & 0.1 & -12 & 58.6 & 310.7 & 5.6 & 90 & 1.4 & 127 & 4.6 & 80 & $\mathrm{c} / \mathrm{cs}$ \\
\hline 28 & 4 & 1985 & 1218 & 42 & 0.1 & -12 & 55.4 & 313.0 & 6.2 & 90 & 1.7 & 119 & 4.8 & 80 & $\mathrm{c} / \mathrm{cs}$ \\
\hline 28 & 4 & 1985 & 1246 & 42 & 0.2 & -12 & 52.0 & 315.6 & 3.5 & 81 & 1.2 & 273 & 4.7 & 84 & $\mathrm{c} / \mathrm{cs}$ \\
\hline 28 & 4 & 1985 & 1254 & 42 & 0.2 & -12 & 51.4 & 316.1 & 3.6 & 84 & 1.2 & 273 & 4.8 & 86 & $\mathrm{c} / \mathrm{cs}$ \\
\hline 28 & 4 & 1985 & 1320 & 42 & 0.4 & -12 & 49.3 & 317.6 & 3.8 & 84 & 1.2 & 273 & 4.9 & 86 & $\mathrm{c} / \mathrm{cs}$ \\
\hline 28 & 4 & 1985 & 1344 & 42 & 0.6 & -12 & 47.3 & 319.1 & 4.0 & 84 & 1.2 & 273 & 5.1 & 86 & $\mathrm{c} / \mathrm{cs}$ \\
\hline 28 & 4 & 1985 & 1414 & 42 & 0.8 & -12 & 44.6 & 321.1 & 3.8 & 84 & 1.2 & 273 & 4.9 & 86 & $\mathrm{c} / \mathrm{cs}$ \\
\hline 28 & 4 & 1985 & 1430 & 42 & 0.9 & -12 & 43.2 & 322.1 & 4.0 & 86 & 1.2 & 273 & 5.2 & 88 & $\mathrm{c} / \mathrm{cs}$ \\
\hline 28 & 4 & 1985 & 1455 & 42 & 1.0 & -12 & 41.0 & 323.8 & 3.5 & 86 & 1.2 & 273 & 4.7 & 88 & $\mathrm{c} / \mathrm{cs}$ \\
\hline 28 & 4 & 1985 & 1456 & 42 & 1.0 & -12 & 40.9 & 323.8 & 5.0 & 350 & 1.2 & 273 & 4.9 & 3 & $\mathrm{c} / \mathrm{cs}$ \\
\hline 28 & 4 & 1985 & 1500 & 42 & 1.3 & -12 & 41.0 & 324.2 & 5.2 & 345 & 1.2 & 273 & 5.0 & 358 & $\mathrm{c} / \mathrm{cs}$ \\
\hline 28 & 4 & 1985 & 1524 & 42 & 3,4 & -12 & 41.7 & 326.3 & 6.6 & 300 & 1.2 & 273 & 5.6 & 305 & $\mathrm{c} / \mathrm{cs}$ \\
\hline 28 & 4 & 1985 & 1529 & 42 & 3.6 & -12 & 42.4 & 326.8 & 7.3 & 297 & 1.2 & 273 & 6.3 & 301 & $\mathrm{c} / \mathrm{cs}$ \\
\hline 28 & 4 & 1985 & 1538 & 42 & 4.1 & -12 & 43.7 & 327.9 & 7.5 & 275 & 1.2 & 273 & 6.3 & 275 & $\mathrm{c} / \mathrm{cs}$ \\
\hline 28 & 4 & 1985 & 1545 & 42 & 4.2 & -12 & 44.9 & 328.8 & 7.8 & 271 & 1.2 & 273 & 6.6 & 271 & $\mathrm{c} / \mathrm{cs}$ \\
\hline 28 & 4 & 1985 & 1556 & 42 & 4.2 & -12 & 46.8 & 330.2 & 6.3 & 274 & 1.2 & 273 & 5.2 & 274 & $\mathrm{c} / \mathrm{cs}$ \\
\hline 28 & 4 & 1985 & 1609 & 42 & 4.3 & -12 & 48.6 & 331.6 & 5.8 & 273 & 1.2 & 273 & 4.6 & 273 & $\mathrm{c} / \mathrm{cs}$ \\
\hline 28 & 4 & 1985 & 1639 & 42 & 4.5 & -12 & 52.5 & 334.5 & 5.8 & 271 & 1.2 & 273 & 4.6 & 271 & $\mathrm{c} / \mathrm{cs}$ \\
\hline 28 & 4 & 1985 & 1709 & 42 & 4.5 & -12 & 56.4 & 337.4 & 5.7 & 270 & 1.2 & 273 & 4.6 & 269 & $\mathrm{c} / \mathrm{cs}$ \\
\hline 28 & 4 & 1985 & 1714 & 42 & 4.5 & -12 & 57.0 & 337.8 & 5.0 & 231 & 1.2 & 273 & 4.3 & 220 & $\mathrm{c} / \mathrm{cs}$ \\
\hline 28 & 4 & 1985 & 1718 & 42 & 4.3 & -12 & 57.4 & 338.2 & 5.0 & 262 & 1.2 & 273 & 3.9 & 259 & $\mathrm{c} / \mathrm{cs}$ \\
\hline 28 & 4 & 1985 & 1720 & 42 & 4.3 & -12 & 57.6 & 338.3 & 4.2 & 300 & 1.2 & 273 & 3.3 & 309 & $\mathrm{c} / \mathrm{cs}$ \\
\hline 28 & 4 & 1985 & 1723 & 42 & 4.4 & -12 & 57.8 & 338.6 & 2.8 & 326 & 1.2 & 273 & 2.3 & 350 & $\mathrm{c} / \mathrm{cs}$ \\
\hline 28 & 4 & 1985 & 1727 & 42 & 4.6 & -12 & 58.0 & 338.7 & 1.9 & 13 & 1.2 & 273 & 2.4 & 42 & $\mathrm{c} / \mathrm{cs}$ \\
\hline 28 & 4 & 1985 & 1730 & 42 & 4.7 & -12 & 58.0 & 338.8 & 2.7 & 67 & 1.2 & 273 & 3.8 & 75 & $\mathrm{c} / \mathrm{cs}$ \\
\hline 28 & 4 & 1985 & 1734 & 42 & 4.7 & -12 & 57.7 & 339.0 & 3.1 & 87 & 1.2 & 273 & 4.2 & 89 & $\mathrm{c} / \mathrm{cs}$ \\
\hline 28 & 4 & 1985 & 1759 & 42 & 4.8 & -12 & 56.0 & 340.3 & 2.7 & 89 & 1.2 & 273 & 3.9 & 90 & $\mathrm{c} / \mathrm{cs}$ \\
\hline 28 & 4 & 1985 & 1818 & 42 & 4.8 & -12 & 54.9 & 341.2 & 1.7 & 81 & 1.2 & 273 & 2.8 & 86 & $\mathrm{c} / \mathrm{cs}$ \\
\hline 28 & 4 & 1985 & 1820 & 42 & 4.8 & -12 & 54.8 & 341.2 & 1.4 & 78 & 1.2 & 273 & 2.5 & 85 & $\mathrm{c} / \mathrm{cs}$ \\
\hline 28 & 4 & 1985 & 2000 & 42 & 5.30 & -12 & 51.80 & 343.5 & 2.5 & 85 & 0.0 & 90 & 2.5 & 85 & S637 \\
\hline 28 & 4 & 1985 & 2000 & 42 & 5.3 & -12 & 51.8 & 343.5 & 0.0 & 90 & 0.0 & 90 & 0.0 & 500 & $\mathrm{c} / \mathrm{cs}$ \\
\hline 29 & 4 & 1985 & 1615 & 42 & 5.30 & -12 & 51.80 & 343.5 & 0.0 & 90 & 0.0 & 90 & 0.0 & 500 & $637 \mathrm{~A}$ \\
\hline 4 & 5 & 1985 & 2105 & 42 & 5.30 & -12 & 51.80 & 343.5 & 1.4 & 127 & 1.4 & 127 & 0.0 & 500 & $637 \mathrm{~A}$ \\
\hline 4 & 5 & 1985 & 2139 & 42 & 4.8 & -12 & 51.0 & 344.3 & 3.3 & 101 & 1.4 & 127 & 2.2 & 85 & $\mathrm{c} / \mathrm{cs}$ \\
\hline 4 & 5 & 1985 & 2140 & 42 & 4.8 & -12 & 50.9 & 344.3 & 4.8 & 172 & 1.4 & 127 & 4.0 & 186 & $\mathrm{c} / \mathrm{cs}$ \\
\hline 4 & 5 & 1985 & 2146 & 42 & 4.3 & -12 & 50.8 & 344.8 & 2.7 & 210 & 1.4 & 127 & 2.8 & 239 & $\mathrm{c} / \mathrm{cs}$ \\
\hline 4 & 5 & 1985 & 2149 & 42 & 4.2 & -12 & 50.9 & 344.9 & 0.7 & 277 & 1.4 & 127 & 2.1 & 297 & $\mathrm{c} / \mathrm{cs}$ \\
\hline 4 & 5 & 1985 & 2153 & 42 & 4.2 & -12 & 50.9 & 345.0 & 1.5 & 25 & 1.4 & 127 & 2.3 & 348 & $\mathrm{c} / \mathrm{cs}$ \\
\hline 4 & 5 & 1985 & 2157 & 42 & 4.3 & -12 & 50.9 & 345.1 & 2.7 & 20 & 1.4 & 127 & 3.3 & 357 & $\mathrm{c} / \mathrm{cs}$ \\
\hline 4 & 5 & 1985 & 2202 & 42 & 4.5 & -12 & 50.8 & 345.3 & 3.9 & 18 & 1.4 & 127 & 4.6 & 1 & $\mathrm{c} / \mathrm{cs}$ \\
\hline 4 & 5 & 1985 & 2217 & 42 & 5.5 & -12 & 50.4 & 346.3 & 3.4 & 15 & 1.4 & 127 & 4.1 & 357 & $\mathrm{c} / \mathrm{cs}$ \\
\hline 4 & 5 & 1985 & 2222 & 42 & 5.7 & -12 & 50.3 & 346.6 & 2.5 & 23 & 1.4 & 127 & 3.2 & 358 & $\mathrm{c} / \mathrm{cs}$ \\
\hline 4 & 5 & 1985 & 2231 & 42 & 6.1 & -12 & 50.1 & 347.0 & 3.6 & 15 & 1.4 & 127 & 4.3 & 358 & $\mathrm{c} / \mathrm{cs}$ \\
\hline 4 & 5 & 1985 & 2259 & 42 & 7.7 & -12 & 49.5 & 348.7 & 4.4 & 61 & 1.4 & 127 & 4.0 & 43 & $\mathrm{c} / \mathrm{cs}$ \\
\hline 4 & 5 & 1985 & 2301 & 42 & 7.8 & -12 & 49.3 & 348.8 & 6.1 & 92 & 1.4 & 127 & 5.0 & 83 & $\mathrm{c} / \mathrm{cs}$ \\
\hline 4 & 5 & 1985 & 2308 & 42 & 7.8 & -12 & 48.4 & 349.5 & 6.2 & 95 & 1.4 & 127 & 5.1 & 86 & $\mathrm{c} / \mathrm{cs}$ \\
\hline 4 & 5 & 1985 & 2334 & 42 & 7.5 & -12 & 44.8 & 352.2 & 6.1 & 96 & 1.4 & 127 & 4.9 & 87 & $\mathrm{c} / \mathrm{cs}$ \\
\hline 5 & 5 & 1985 & 0001 & 42 & 7.3 & -12 & 41.1 & 354.9 & 6.1 & 95 & 1.4 & 127 & 5.0 & 87 & $\mathrm{c} / \mathrm{cs}$ \\
\hline 5 & 5 & 1985 & 0017 & 42 & 7.1 & -12 & 38.9 & 356.5 & 5.9 & 97 & 1.4 & 127 & 4.8 & 89 & $\mathrm{c} / \mathrm{cs}$ \\
\hline 5 & 5 & 1985 & 0047 & 42 & 6.7 & -12 & 35.0 & 359.5 & 5.8 & 95 & 1.4 & 127 & 4.7 & 86 & $\mathrm{c} / \mathrm{cs}$ \\
\hline 5 & 5 & 1985 & 0105 & 42 & 6.6 & -12 & 32.6 & 361.2 & 5.9 & 97 & 1.4 & 127 & 4.7 & 88 & $\mathrm{c} / \mathrm{cs}$ \\
\hline 5 & 5 & 1985 & 0112 & 42 & 6.5 & -12 & 31.7 & 361.9 & 5.9 & 97 & 1.4 & 127 & 4.7 & 89 & $\mathrm{c} / \mathrm{cs}$ \\
\hline 5 & 5 & 1985 & 0118 & 42 & 6.44 & -12 & 30.91 & 362.5 & 5.5 & 87 & 0.8 & 74 & 4.7 & 89 & satl \\
\hline 5 & 5 & 1985 & 0210 & 42 & 6.7 & -12 & 24.5 & 367.3 & 5.4 & 75 & 0.8 & 74 & 4.6 & 75 & $\mathrm{c} / \mathrm{cs}$ \\
\hline 5 & 5 & 1985 & 0219 & 42 & 6.9 & -12 & 23.4 & 368.1 & 5.4 & 71 & 0.8 & 74 & 4.6 & 70 & $\mathrm{c} / \mathrm{cs}$ \\
\hline 5 & 5 & 1985 & 0239 & 42 & 7.5 & -12 & 21.1 & 369.9 & 5.0 & 67 & 0.8 & 74 & 4.2 & 65 & $\mathrm{c} / \mathrm{cs}$ \\
\hline 5 & 5 & 1985 & 0251 & 42 & 7.9 & -12 & 19.9 & 370.9 & 5.3 & 71 & 0.8 & 74 & 4.5 & 70 & $\mathrm{c} / \mathrm{cs}$ \\
\hline 5 & 5 & 1985 & 0305 & 42 & 8.3 & -12 & 18.3 & 372.2 & 5.2 & 66 & 0.8 & 74 & 4.4 & 65 & $\mathrm{c} / \mathrm{cs}$ \\
\hline 5 & 5 & 1985 & 0314 & 42 & 8.6 & -12 & 17.3 & 372.9 & 5.2 & 67 & 0.8 & 74 & 4.4 & 66 & $\mathrm{c} / \mathrm{cs}$ \\
\hline 5 & 5 & 1985 & 0319 & 42 & 8.8 & -12 & 16.8 & 373.4 & 5.4 & 76 & 0.8 & 74 & 4.6 & 76 & $\mathrm{c} / \mathrm{cs}$ \\
\hline 5 & 5 & 1985 & 0330 & 42 & 9.0 & -12 & 15.5 & 374.4 & 5.4 & 77 & 0.8 & 74 & 4.6 & 77 & $\mathrm{c} / \mathrm{cs}$ \\
\hline 5 & 5 & 1985 & 0334 & 42 & 9.1 & -12 & 15.0 & 374.7 & 5.3 & 72 & 0.8 & 74 & 4.5 & 71 & $\mathrm{c} / \mathrm{cs}$ \\
\hline 5 & 5 & 1985 & 0343 & 42 & 9.4 & -12 & 14.0 & 375.5 & 5.2 & 72 & 0.8 & 74 & 4.3 & 72 & $\mathrm{c} / \mathrm{cs}$ \\
\hline 5 & 5 & 1985 & 0347 & 42 & 9.5 & -12 & 13.6 & 375.9 & 5.3 & 85 & 0.8 & 74 & 4.5 & 87 & $\mathrm{c} / \mathrm{cs}$ \\
\hline 5 & 5 & 1985 & 0352 & 42 & 9.5 & -12 & 13.0 & 376.3 & 5.7 & 79 & 0.8 & 74 & 4.9 & 80 & $\mathrm{c} / \mathrm{cs}$ \\
\hline 5 & 5 & 1985 & 0358 & 42 & 9.63 & -12 & 12.21 & 376.9 & 3.3 & 86 & 1.7 & 247 & 4.9 & 80 & satl \\
\hline 5 & 5 & 1985 & 0411 & 42 & 9.7 & -12 & 11.3 & 377.6 & 3.2 & 88 & 1.7 & 247 & 4.8 & 81 & $\mathrm{c} / \mathrm{cs}$ \\
\hline 5 & 5 & 1985 & 0427 & 42 & 9.7 & -12 & 10.1 & 378.4 & 2.9 & 112 & 1.7 & 247 & 4.3 & 96 & $\mathrm{c} / \mathrm{cs}$ \\
\hline 5 & 5 & 1985 & 0428 & 42 & 9.7 & -12 & 10.0 & 378.5 & 3.9 & 173 & 1.7 & 247 & 3.8 & 148 & $\mathrm{c} / \mathrm{cs}$ \\
\hline 5 & 5 & 1985 & 0430 & 42 & 9.6 & -12 & 10.0 & 378.6 & 5.5 & 206 & 1.7 & 247 & 4.4 & 191 & $\mathrm{c} / \mathrm{cs}$ \\
\hline 5 & 5 & 1985 & 0433 & 42 & 9.3 & -12 & 10.2 & 378.9 & 5.6 & 228 & 1.7 & 247 & 4.1 & 220 & $\mathrm{c} / \mathrm{cs}$ \\
\hline 5 & 5 & 1985 & 0435 & 42 & 9.2 & -12 & 10.4 & 379.1 & 5.1 & 250 & 1.7 & 247 & 3.4 & 252 & $\mathrm{c} / \mathrm{cs}$ \\
\hline 5 & 5 & 1985 & 0438 & 42 & 9.1 & -12 & 10.7 & 379.3 & 5.3 & 266 & 1.7 & 247 & 3.7 & 274 & $\mathrm{c} / \mathrm{cs}$ \\
\hline 5 & 5 & 1985 & 0457 & 42 & 9.0 & -12 & 12.9 & 381.0 & 4.8 & 265 & 1.7 & 247 & 3.3 & 274 & $\mathrm{c} / \mathrm{cs}$ \\
\hline 5 & 5 & 1985 & 0516 & 42 & 8.8 & -12 & 15.0 & 382.5 & 4.4 & 258 & 1.7 & 247 & 2.8 & 265 & $\mathrm{c} / \mathrm{cs}$ \\
\hline 5 & 5 & 1985 & 0520 & 42 & 8.8 & -12 & 15.4 & 382.8 & 4.9 & 218 & 1.7 & 247 & 3.6 & 205 & $\mathrm{c} / \mathrm{cs}$ \\
\hline 5 & 5 & 1985 & 0522 & 42 & 8.7 & -12 & 15.5 & 383.0 & 4.4 & 171 & 1.7 & 247 & 4.3 & 149 & $\mathrm{c} / \mathrm{cs}$ \\
\hline 5 & 5 & 1985 & 0524 & 42 & 8.5 & -12 & 15.5 & 383.1 & 2.8 & 105 & 1.7 & 247 & 4.3 & 91 & $\mathrm{c} / \mathrm{cs}$ \\
\hline 5 & 5 & 1985 & 0526 & 42 & 8.5 & -12 & 15.3 & 383.2 & 2.7 & 65 & 1.7 & 247 & 4.3 & 66 & $\mathrm{c} / \mathrm{cs}$ \\
\hline
\end{tabular}


Table 1 (continued).

\begin{tabular}{|c|c|c|c|c|c|c|c|c|c|c|c|c|c|c|c|}
\hline \multirow[b]{3}{*}{ Day } & & & & & & & & & & ual & & & $\begin{array}{r}\mathrm{D} \\
\text { recl }\end{array}$ & $\begin{array}{l}\text { ad } \\
\text { ning }\end{array}$ & \\
\hline & & & & & & long & & & speed & course & speed & hed & speed & course & \\
\hline & Month & Year & Time $^{\mathrm{a}}$ & deg & $\min$ & deg & $\min$ & Dist & (kt) & (deg) & $(\mathrm{kt})$ & (deg) & (kt) & (deg) & Comments $^{b}$ \\
\hline 5 & 5 & 1985 & 0532 & 42 & 8.6 & -12 & 15.0 & 383.5 & 3.2 & 73 & 1.7 & 247 & 4.8 & 71 & $\mathrm{c} / \mathrm{cs}$ \\
\hline 5 & 5 & 1985 & 0541 & 42 & 8.7 & -12 & 14.4 & 384.0 & 2.9 & 37 & 1.7 & 247 & 4.4 & 48 & $\mathrm{c} / \mathrm{cs}$ \\
\hline 5 & 5 & 1985 & 0543 & 42 & 8.8 & -12 & 14.3 & 384.1 & 2.8 & 305 & 1.7 & 247 & 2.4 & 342 & $\mathrm{c} / \mathrm{cs}$ \\
\hline 5 & 5 & 1985 & 0546 & 42 & 8.9 & -12 & 14.5 & 384.2 & 4.2 & 268 & 1.7 & 247 & 2.7 & 281 & $\mathrm{c} / \mathrm{cs}$ \\
\hline 5 & 5 & 1985 & 0553 & 42 & 8.9 & -12 & 15.1 & 384.7 & 4.7 & 267 & 1.7 & 247 & 3.2 & 277 & $\mathrm{c} / \mathrm{cs}$ \\
\hline 5 & 5 & 1985 & 0605 & 42 & 8.8 & -12 & 16.4 & 385.6 & 4.9 & 227 & 1.7 & 247 & 3.4 & 218 & $\mathrm{c} / \mathrm{cs}$ \\
\hline 5 & 5 & 1985 & 0607 & 42 & 8.7 & -12 & 16.5 & 385.8 & 3.7 & 168 & 1.7 & 247 & 3.8 & 143 & $\mathrm{c} / \mathrm{cs}$ \\
\hline 5 & 5 & 1985 & 0609 & 42 & 8.6 & -12 & 16.5 & 385.9 & 2.2 & 86 & 1.7 & 247 & 3.8 & 78 & $\mathrm{c} / \mathrm{cs}$ \\
\hline 5 & 5 & 1985 & 0612 & 42 & 8.6 & -12 & 16.4 & 386.0 & 2.9 & 79 & 1.7 & 247 & 4.5 & 75 & $\mathrm{c} / \mathrm{cs}$ \\
\hline 5 & 5 & 1985 & 0619 & 42 & 8.7 & -12 & 15.9 & 386.4 & 3.4 & 79 & 1.7 & 247 & 5.0 & 75 & $\mathrm{c} / \mathrm{cs}$ \\
\hline 5 & 5 & 1985 & 0624 & 42 & 8.7 & -12 & 15.6 & 386.6 & 3.3 & 80 & 1.7 & 247 & 4.9 & 76 & $\mathrm{c} / \mathrm{cs}$ \\
\hline 5 & 5 & 1985 & 0715 & 42 & 9.20 & -12 & 11.80 & 389.5 & 4.9 & 76 & 0.0 & 90 & 4.9 & 76 & S638 \\
\hline 5 & 5 & 1985 & 0715 & 42 & 9.2 & -12 & 11.8 & 389.5 & 0.0 & 90 & 0.0 & 90 & 0.0 & 500 & $\mathrm{c} / \mathrm{cs}$ \\
\hline 5 & 5 & 1985 & 0845 & 42 & 9.20 & -12 & 11.80 & 389.5 & 0.0 & 90 & 0.0 & 90 & 0.0 & 500 & $638 \mathrm{~A}$ \\
\hline 6 & 5 & 1985 & 0015 & 42 & 9.20 & -12 & 11.80 & 389.5 & 0.0 & 90 & 0.0 & 90 & 0.0 & 500 & $638 \mathrm{~A}$ \\
\hline 6 & 5 & 1985 & 0145 & 42 & 9.20 & -12 & 11.80 & 389.5 & 0.0 & 90 & 0.0 & 90 & 0.0 & 500 & 638B \\
\hline 12 & 5 & 1985 & 1329 & 42 & 9.20 & -12 & 11.80 & 389.5 & 0.0 & 90 & 0.0 & 90 & 0.0 & 500 & $638 \mathrm{~B}$ \\
\hline 12 & 5 & 1985 & 2145 & 42 & 9.20 & -12 & 11.80 & 389.5 & 0.0 & 255 & 0.0 & 255 & 0.0 & 500 & $638 \mathrm{C}$ \\
\hline 23 & 5 & 1985 & 2000 & 42 & 8.60 & -12 & 14.90 & 391.8 & 0.0 & 90 & 0.0 & 90 & 0.0 & 500 & $639 A$ \\
\hline 25 & 5 & 1985 & 1245 & 42 & 8.60 & -12 & 14.90 & 391.8 & 0.0 & 270 & 0.0 & 270 & 0.0 & 500 & $639 A$ \\
\hline 26 & 5 & 1985 & 1340 & 42 & 8.60 & -12 & 15.00 & 391.9 & 0.0 & 90 & 0.0 & 90 & 0.0 & 500 & 639B \\
\hline 27 & 5 & 1985 & 0545 & 42 & 8.60 & -12 & 15.00 & 391.9 & 0.0 & 270 & 0.0 & 270 & 0.0 & 500 & 639B \\
\hline 27 & 5 & 1985 & 1230 & 42 & 8.60 & -12 & 15.10 & 392.0 & 0.0 & 90 & 0.0 & 90 & 0.0 & 500 & $639 \mathrm{C}$ \\
\hline 27 & 5 & 1985 & 2230 & 42 & 8.60 & -12 & 15.10 & 392.0 & 0.1 & 270 & 0.1 & 270 & 0.0 & 500 & $639 \mathrm{C}$ \\
\hline 28 & 5 & 1985 & 0000 & 42 & 8.60 & -12 & 15.30 & 392.1 & 0.0 & 90 & 0.0 & 90 & 0.0 & 500 & 639D \\
\hline 1 & 6 & 1985 & 0130 & 42 & 8.60 & -12 & 15.30 & 392.1 & 0.1 & 76 & 0.1 & 76 & 0.0 & 500 & 639D \\
\hline 1 & 6 & 1985 & 2307 & 42 & 9.04 & -12 & 12.85 & 394.0 & 0.0 & 78 & 0.0 & 78 & 0.0 & 500 & $638 \mathrm{C}$ \\
\hline 3 & 6 & 1985 & 0945 & 42 & 9.20 & -12 & 11.80 & 394.8 & 0.3 & 253 & 0.3 & 253 & 0.0 & 500 & $638 C$ \\
\hline 3 & 6 & 1985 & 2027 & 42 & 8.4 & -12 & 15.6 & 397.7 & 4.4 & 75 & 0.3 & 253 & 4.7 & 75 & $\mathrm{c} / \mathrm{cs}$ \\
\hline 3 & 6 & 1985 & 2028 & 42 & 8.4 & -12 & 15.5 & 397.8 & 4.5 & 202 & 0.3 & 253 & 4.4 & 199 & $\mathrm{c} / \mathrm{cs}$ \\
\hline 3 & 6 & 1985 & 2034 & 42 & 8.0 & -12 & 15.7 & 398.3 & 4.9 & 202 & 0.3 & 253 & 4.8 & 199 & $\mathrm{c} / \mathrm{cs}$ \\
\hline 3 & 6 & 1985 & 2045 & 42 & 7.1 & -12 & 16.2 & 399.2 & 5.5 & 201 & 0.3 & 253 & 5.3 & 199 & $\mathrm{c} / \mathrm{cs}$ \\
\hline 3 & 6 & 1985 & 2106 & 42 & 5.32 & -12 & 17.13 & 401.1 & 5.0 & 212 & 1.2 & 309 & 5.3 & 199 & satl \\
\hline 3 & 6 & 1985 & 2115 & 42 & 4.7 & -12 & 17.7 & 401.9 & 5.0 & 211 & 1.2 & 309 & 5.3 & 198 & $\mathrm{c} / \mathrm{cs}$ \\
\hline 3 & 6 & 1985 & 2131 & 42 & 3.5 & -12 & 18.6 & 403.2 & 5.1 & 209 & 1.2 & 309 & 5.4 & 196 & $\mathrm{c} / \mathrm{cs}$ \\
\hline 3 & 6 & 1985 & 2138 & 42 & 3.0 & -12 & 19.0 & 403.8 & 4.9 & 201 & 1.2 & 309 & 5.4 & 189 & $\mathrm{c} / \mathrm{cs}$ \\
\hline 3 & 6 & 1985 & 2144 & 42 & 2.57 & -12 & 19.20 & 404.3 & 6.7 & 214 & 3.0 & 265 & 5.4 & 189 & satl \\
\hline 3 & 6 & 1985 & 2208 & 42 & 0.3 & -12 & 21.2 & 407.0 & 7.9 & 268 & 3.0 & 265 & 4.9 & 270 & $\mathrm{c} / \mathrm{cs}$ \\
\hline 3 & 6 & 1985 & 2211 & 42 & 0.3 & -12 & 21.8 & 407.3 & 8.3 & 268 & 3.0 & 265 & 5.3 & 270 & $\mathrm{c} / \mathrm{cs}$ \\
\hline 3 & 6 & 1985 & 2241 & 42 & 0.2 & -12 & 27.4 & 411.5 & 8.3 & 268 & 3.0 & 265 & 5.3 & 270 & $\mathrm{c} / \mathrm{cs}$ \\
\hline 3 & 6 & 1985 & 2256 & 42 & 0.12 & -12 & 30.15 & 413.6 & 6.5 & 272 & 1.2 & 281 & 5.3 & 270 & satl \\
\hline 3 & 6 & 1985 & 2315 & 42 & 0.2 & -12 & 32.9 & 415.6 & 6.2 & 276 & 1.2 & 281 & 5.0 & 275 & $\mathrm{c} / \mathrm{cs}$ \\
\hline 3 & 6 & 1985 & 2317 & 42 & 0.2 & -12 & 33.2 & 415.8 & 6.5 & 287 & 1.2 & 281 & 5.4 & 289 & $\mathrm{c} / \mathrm{cs}$ \\
\hline 3 & 6 & 1985 & 2326 & 42 & 0.5 & -12 & 34.4 & 416.8 & 6.0 & 287 & 1.2 & 281 & 4.8 & 289 & $\mathrm{c} / \mathrm{cs}$ \\
\hline 3 & 6 & 1985 & 2334 & 42 & 0.7 & -12 & 35.5 & 417.6 & 5.9 & 191 & 1.2 & 281 & 6.0 & 180 & $\mathrm{c} / \mathrm{cs}$ \\
\hline 3 & 6 & 1985 & 2340 & 42 & 0.2 & -12 & 35.6 & 418.2 & 5.1 & 88 & 1.2 & 281 & 6.2 & 90 & $\mathrm{c} / \mathrm{cs}$ \\
\hline 4 & 6 & 1985 & 0040 & 42 & 0.4 & -12 & 28.8 & 423.2 & 6.5 & 288 & 1.2 & 281 & 5.3 & 290 & $\mathrm{c} / \mathrm{cs}$ \\
\hline 4 & 6 & 1985 & 0055 & 42 & 0.9 & -12 & 30.9 & 424.9 & 6.7 & 288 & 1.2 & 281 & 5.5 & 290 & $\mathrm{c} / \mathrm{cs}$ \\
\hline 4 & 6 & 1985 & 0100 & 42 & 1.1 & -12 & 31.6 & 425.4 & 1.2 & 281 & 1.2 & 281 & 0.0 & 500 & $\mathrm{c} / \mathrm{cs}$ \\
\hline 4 & 6 & 1985 & 0136 & 42 & 1.20 & -12 & 32.56 & 426.1 & 0.4 & 100 & 0.4 & 100 & 0.0 & 500 & satl \\
\hline 4 & 6 & 1985 & 1015 & 42 & 0.60 & -12 & 27.80 & 429.7 & 0.0 & 90 & 0.0 & 90 & 0.0 & 500 & $640 \mathrm{~A}$ \\
\hline 6 & 6 & 1985 & 0100 & 42 & 0.60 & -12 & 27.80 & 429.7 & 1.1 & 28 & 1.1 & 28 & 0.0 & 500 & $640 \mathrm{~A}$ \\
\hline 6 & 6 & 1985 & 0240 & 42 & 2.3 & -12 & 26.6 & 431.6 & 5.1 & 286 & 1.1 & 28 & 5.5 & 274 & $\mathrm{c} / \mathrm{cs}$ \\
\hline 6 & 6 & 1985 & 0241 & 42 & 2.3 & -12 & 26.7 & 431.7 & 5.1 & 8 & 1.1 & 28 & 4.1 & 3 & $\mathrm{c} / \mathrm{cs}$ \\
\hline 6 & 6 & 1985 & 0248 & 42 & 2.9 & -12 & 26.6 & 432.3 & 6.0 & 4 & 1.1 & 28 & 5.0 & 359 & $\mathrm{c} / \mathrm{cs}$ \\
\hline 6 & 6 & 1985 & 0256 & 42 & 3.7 & -12 & 26.5 & 433.1 & 5.9 & 355 & 1.1 & 28 & 5.0 & 348 & $\mathrm{c} / \mathrm{cs}$ \\
\hline 6 & 6 & 1985 & 0326 & 42 & 6.6 & -12 & 26.9 & 436.1 & 6.0 & 355 & 1.1 & 28 & 5.1 & 348 & $\mathrm{c} / \mathrm{cs}$ \\
\hline 6 & 6 & 1985 & 0342 & 42 & 8.2 & -12 & 27.0 & 437.7 & 5.5 & 6 & 1.1 & 28 & 4.4 & 1 & $\mathrm{c} / \mathrm{cs}$ \\
\hline 6 & 6 & 1985 & 0343 & 42 & 8.3 & -12 & 27.0 & 437.8 & 5.5 & 33 & 1.1 & 28 & 4.3 & 34 & $\mathrm{c} / \mathrm{cs}$ \\
\hline 6 & 6 & 1985 & 0344 & 42 & 8.4 & -12 & 27.0 & 437.9 & 4.9 & 75 & 1.1 & 28 & 4.3 & 86 & $\mathrm{c} / \mathrm{cs}$ \\
\hline 6 & 6 & 1985 & 0347 & 42 & 8.5 & -12 & 26.6 & 438.1 & 6.0 & 83 & 1.1 & 28 & 5.4 & 93 & $\mathrm{c} / \mathrm{cs}$ \\
\hline 6 & 6 & 1985 & 0358 & 42 & 8.6 & -12 & 25.2 & 439.2 & 6.0 & 83 & 1.1 & 28 & 5.4 & 93 & $\mathrm{c} / \mathrm{cs}$ \\
\hline 6 & 6 & 1985 & 0426 & 42 & 8.95 & -12 & 21.43 & 442.0 & 6.6 & 90 & 1.2 & 78 & 5.4 & 93 & satl \\
\hline 6 & 6 & 1985 & 0428 & 42 & 8.9 & -12 & 21.1 & 442.2 & 6.7 & 90 & 1.2 & 78 & 5.5 & 93 & $\mathrm{c} / \mathrm{cs}$ \\
\hline 6 & 6 & 1985 & 0445 & 42 & 8.9 & -12 & 18.6 & 444.1 & 6.5 & 98 & 1.2 & 78 & 5.3 & 103 & $\mathrm{c} / \mathrm{cs}$ \\
\hline 6 & 6 & 1985 & 0448 & 42 & 8.89 & -12 & 18.13 & 444.5 & 5.9 & 100 & 0.6 & 73 & 5.3 & 103 & satl \\
\hline 6 & 6 & 1985 & 0502 & 42 & 8.7 & -12 & 16.3 & 445.8 & 6.0 & 101 & 0.6 & 73 & 5.4 & 104 & $\mathrm{c} / \mathrm{cs}$ \\
\hline 6 & 6 & 1985 & 0520 & 42 & 8.3 & -12 & 13.9 & 447.6 & 0.6 & 73 & 0.6 & 73 & 0.0 & 500 & $\mathrm{c} / \mathrm{cs}$ \\
\hline 6 & 6 & 1985 & 0626 & 42 & 8.52 & -12 & 13.04 & 448.3 & 0.3 & 273 & 0.3 & 273 & 0.0 & 500 & satl \\
\hline 6 & 6 & 1985 & 1208 & 42 & 8.60 & -12 & 15.40 & 450.1 & 0.0 & 90 & 0.0 & 90 & 0.0 & 500 & $639 \mathrm{E}$ \\
\hline 7 & 6 & 1985 & 1340 & 42 & 8.60 & -12 & 15.40 & 450.1 & 0.1 & 270 & 0.1 & 270 & 0.0 & 500 & $639 \mathrm{E}$ \\
\hline 7 & 6 & 1985 & 1450 & 42 & 8.60 & -12 & 15.50 & 450.1 & 0.0 & 90 & 0.0 & 90 & 0.0 & 500 & $639 \mathrm{~F}$ \\
\hline 8 & 6 & 1985 & 0200 & 42 & 8.60 & -12 & 15.50 & 450.1 & 0.2 & 78 & 0.2 & 78 & 0.0 & 500 & $639 \mathrm{~F}$ \\
\hline 8 & 6 & 1985 & 1406 & 42 & 9.19 & -12 & 11.76 & 453.0 & 1.7 & 270 & 1.7 & 270 & 0.0 & 500 & satl \\
\hline 8 & 6 & 1985 & 1736 & 42 & 9.24 & -12 & 19.92 & 459.0 & 0.5 & 89 & 0.5 & 89 & 0.0 & 500 & satl \\
\hline 9 & 6 & 1985 & 0630 & 42 & 9.30 & -12 & 10.90 & 465.7 & 0.0 & 90 & 0.0 & 90 & 0.0 & 500 & $641 \mathrm{~A}$ \\
\hline 15 & 6 & 1985 & 1200 & 42 & 9.30 & -12 & 10.90 & 465.7 & 0.0 & 90 & 0.0 & 0 & 0.0 & 500 & $641 \mathrm{C}$ \\
\hline
\end{tabular}

a Universal Coordinated Time.

${ }^{b}$ Satl = satellite navigation; $\mathrm{c} / \mathrm{cs}=$ change of course. 


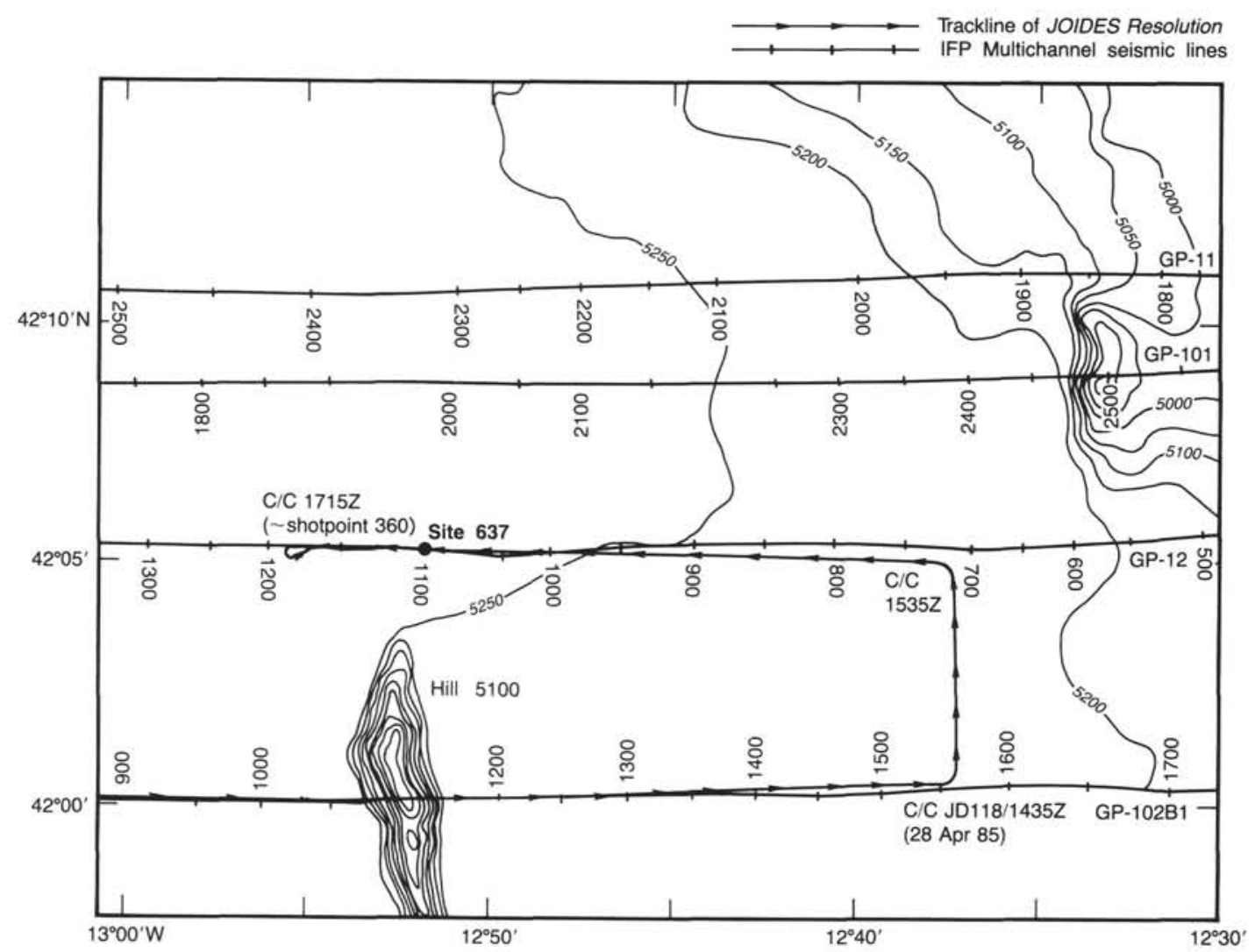

Figure 2. Detailed navigation plot showing track of JOIDES Resolution on approach to Site 637 (see Site 637 chapter, this volume). Course changes are marked on unprocessed analog and processed digital seismic profiles (Figs. 6 and 7). Sea Beam bathymetry courtesy of J. C. Sibuet (see Sibuet et al., this volume).

Five seismic lines were collected during the cruise, as follows:

1. Seismic line 1 was collected en route to Site 637 , the first site visited during Leg 103 . The EDO 2 record of the unprocessed analog data is shown in Figure 6 (from Julian Day $117 / 1400 \mathrm{Z}$ to Julian Day 118/1813Z). The Versatec plot of the part of these data that were reprocessed is shown in Figure 7 (approximately from Julian Day 118/1645Z to 118/1810Z). Navigation on approach to Site 637 is shown in Figure 2; course and speed changes are indicated on both the EDO 2 records and the Versatec plot.

2. Seismic line 2 was collected during the transit from Site 637 to Site 638 . The EDO 2 record of the unprocessed analog data is shown in Figure 8 (from Julian Day 124/2135Z to Julian Day 125/0625Z). The Versatec plot of the part of these data near Site 638 that were reprocessed is shown in Figure 9 (Shotpoints 1352-2621; from Julian Day 125/0210Z to 125/0624Z). Navigation on approach to Site 638 is shown in Figure 3; course and speed changes are indicated on both the EDO 2 record and the Versatec plot.

3. Seismic line 3 was collected during the transit between Site 638 and Site 639. The EDO 2 record is shown in Figure 10 (from Julian Day $143 / 0646 Z$ to $143 / 0958 Z$ ). The data were not reprocessed, so no Versatec plots are available. Navigation on ap- proach to Site 639 is shown in Figure 4; course changes are indicated on the EDO profile.

4. Seismic line 4 was collected during the transit from Site 638 to Site 640 , which occurred after logging Hole $638 \mathrm{C}$. The EDO 2 record is shown in Figure 11 (from Julian Day 154/ $2032 Z$ to $155 / 0122 Z$ ). The Versatec plot of the reprocessed data is shown in Figure 12 (shotpoints 0-1472; from Julian Day 154/ $2027 \mathrm{Z}$ to $155 / 0121 \mathrm{Z}$ ). Navigation on approach to Site 640 is shown in Figure 5; course and speed changes are indicated on both the EDO 2 record and the Versatec plot.

5. Seismic line 5 was collected during the transit from Site 640 to Site 639 . We returned to Site 639 to drill Holes $639 \mathrm{E}$ and 639F (see Site 639 chapter, this volume). The EDO 2 record is shown in Figure 13 (from Julian Day 157/0240Z to 157/0525Z). The Versatec plot of the reprocessed data is shown in Figure 14 (shotpoints 0-800; from Julian Day 157/0240Z to $157 / 0520 Z$ ). Course changes are indicated on both the EDO 2 record and the Versatec plot. Navigation on approach to Site 639 is shown in Figure 1B.

Seismic data were not collected after leaving Site 639 during the transit to Site 641 because of the short transit time and distances involved. In addition, the $3 \mathrm{hr}$ of seismic data collected after leaving Site 641 were not reprocessed and are not presented here. 


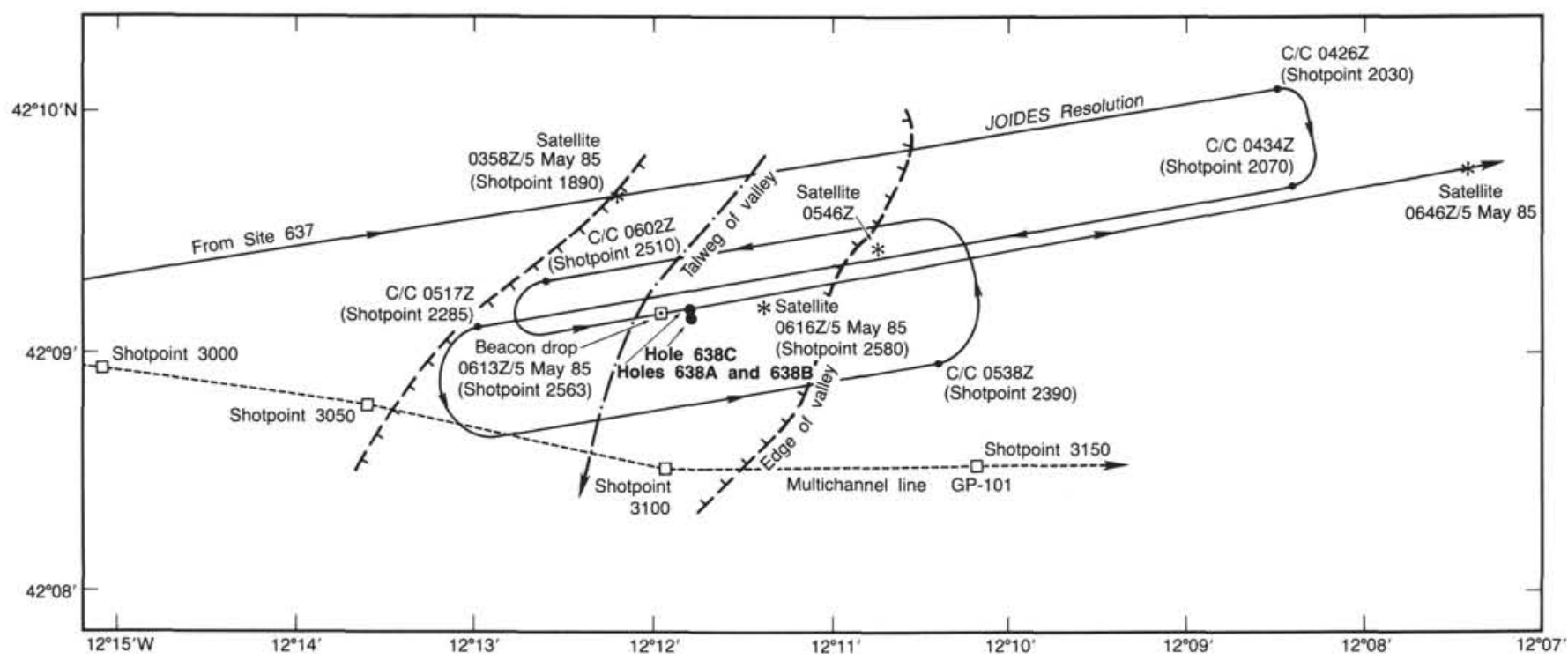

Figure 3. Detailed navigation plot showing track of JOIDES Resolution on approach to Site 638 (see Site 638 chapter, this volume). Course changes are marked on unprocessed analog and processed digital seismic profiles (Figs. 8 and 9). Location of edge of valley determined from shipboard seismic data.

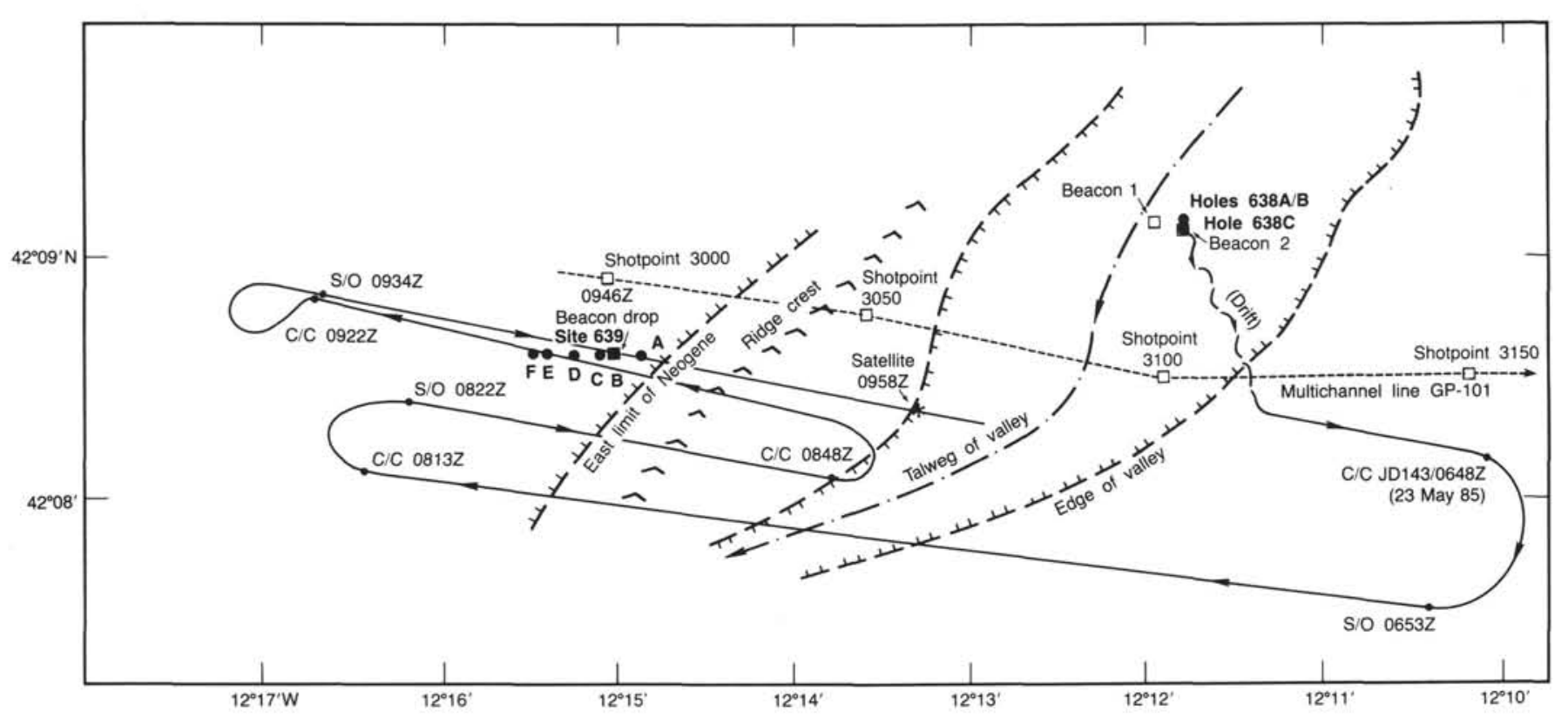

Figure 4. Detailed navigation plot showing track of JOIDES Resolution on approach to Site 639 (see Site 639 chapter, this volume). Course changes are marked on unprocessed analog seismic profile (Fig. 10). Location of edge of valley determined from shipboard seismic data. 


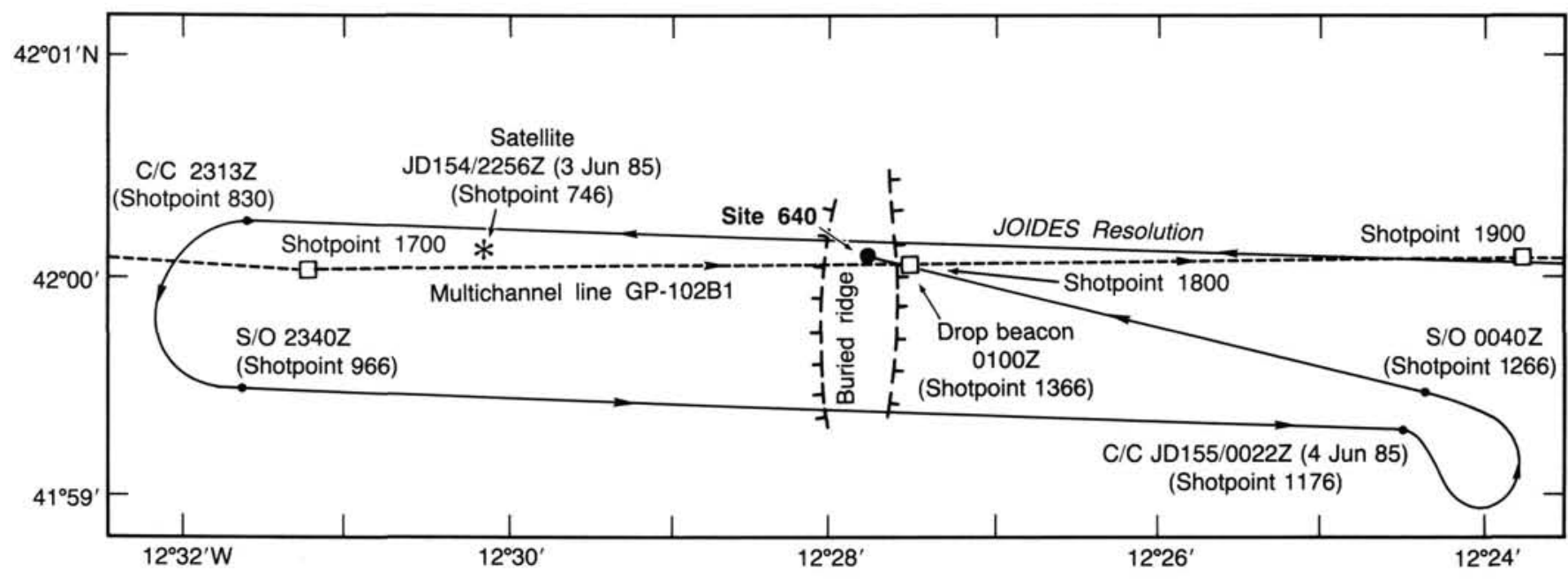

Figure 5. Detailed navigation plot showing track of JOIDES Resolution on approach to Site 640 (see Site 640 chapter, this volume). Course changes are marked on unprocessed analog and processed digital seismic profiles (Figs. 11 and 12). Location of buried ridge determined from shipboard seismic data.

Table 2. Seismic data real-time recording parameters. ${ }^{\mathrm{a}}$

\begin{tabular}{|c|c|c|c|c|c|}
\hline & Line 1 & Line 2 & Line 3 & Line 4 & Line 5 \\
\hline Start at: & $\begin{array}{l}40^{\circ} 30^{\prime} \mathrm{N} \\
17^{\circ} 13^{\prime} \mathrm{W}\end{array}$ & Site 637 & Site 638 & Site 638 & Site 640 \\
\hline $\begin{array}{l}\text { End at: } \\
\text { Source: }\end{array}$ & $\begin{array}{l}\text { Site } 637 \\
\text { Two } 80 \text { in. } \\
\text { water guns }\end{array}$ & $\begin{array}{l}\text { Site } 638 \\
\text { Two } 80 \text { in. }^{3} \\
\text { water guns }\end{array}$ & $\begin{array}{l}\text { Site } 639 \\
\text { Two } 80 \text { in. }^{3} \\
\text { water guns }\end{array}$ & $\begin{array}{l}\text { Site } 640 \\
\text { Two } 80 \text { in. }^{3} \\
\text { water guns }\end{array}$ & $\begin{array}{l}\text { Site } 639 \\
\text { Two } 80 \text { in. }^{3} \\
\text { water guns }\end{array}$ \\
\hline Streamer: & Port & Port & Port & Port & Port \\
\hline \multicolumn{6}{|l|}{ EDO I: } \\
\hline $\begin{array}{l}\text { High cut: } \\
\text { Low cut: } \\
\text { Gain: }\end{array}$ & $\begin{array}{l}200 \text { to } 300 \mathrm{~Hz} \\
70 \text { to } 80 \mathrm{~Hz}\end{array}$ & $\begin{array}{l}200 \mathrm{~Hz} \\
70 \text { to } 80 \mathrm{~Hz}\end{array}$ & $\begin{array}{l}200 \mathrm{~Hz} \\
60 \mathrm{~Hz}\end{array}$ & $\begin{array}{l}200 \mathrm{~Hz} \\
60 \text { to } 70 \mathrm{~Hz}\end{array}$ & $\begin{array}{l}200 \mathrm{~Hz} \\
70 \mathrm{~Hz}\end{array}$ \\
\hline $\begin{array}{l}\text { Amp: } \\
\text { Recorder: }\end{array}$ & $\begin{array}{l}80 \text { to } 85 \mathrm{~dB} \\
\text { Full }\end{array}$ & $\begin{array}{l}80 \text { to } 90 \mathrm{~dB} \\
\text { Full }\end{array}$ & $\begin{array}{l}90 \mathrm{~dB} \\
\text { Full }\end{array}$ & $\begin{array}{l}90 \mathrm{~dB} \\
\text { Full }\end{array}$ & $\begin{array}{l}90 \mathrm{~dB} \\
\text { Full }\end{array}$ \\
\hline \multicolumn{6}{|l|}{$E D O$ 2: } \\
\hline $\begin{array}{l}\text { High cut: } \\
\text { Low cut: } \\
\text { Gain: }\end{array}$ & $\begin{array}{l}200 \text { to } 320 \mathrm{~Hz} \\
80 \mathrm{~Hz}\end{array}$ & $\begin{array}{l}200 \mathrm{~Hz} \\
60 \text { to } 80 \mathrm{~Hz}\end{array}$ & $\begin{array}{l}200 \mathrm{~Hz} \\
60 \mathrm{~Hz}\end{array}$ & $\begin{array}{l}200 \mathrm{~Hz} \\
60 \text { to } 70 \mathrm{~Hz}\end{array}$ & $\begin{array}{l}200 \mathrm{~Hz} \\
70 \mathrm{~Hz}\end{array}$ \\
\hline $\begin{array}{c}\text { Amp: } \\
\text { Recorder: }\end{array}$ & $\begin{array}{l}80 \mathrm{~dB} \\
\text { Full }\end{array}$ & $\begin{array}{l}80 \text { to } 90 \mathrm{~dB} \\
\text { Full }\end{array}$ & $\begin{array}{l}90 \mathrm{~dB} \\
\text { Full }\end{array}$ & $\begin{array}{l}90 \mathrm{~dB} \\
\text { Full }\end{array}$ & $\begin{array}{l}90 \mathrm{~dB} \\
\text { Full }\end{array}$ \\
\hline
\end{tabular}

${ }^{a}$ Unprocessed analog EDO 2 seismic data from lines 1 through 5 are shown in Figures 6, 8, 10, 11 , and 13 , respectively.

Table 3. Seismic-data-processing and -reprocessing parameters. ${ }^{\mathrm{a}}$

\begin{tabular}{lccccc}
\hline & Line 1 & Line 2 & Line 3 & Line 4 & Line 5 \\
\hline Data window: & 6000 to & 5000 to & - & 6000 to & 6000 to \\
& $8000 \mathrm{~ms}$ & $8000 \mathrm{~ms}$ & & $8000 \mathrm{~ms}$ & $8000 \mathrm{~ms}$ \\
AGC: & & & & & \\
$\quad$ Response time: & $500 \mathrm{~ms}$ & $500 \mathrm{~ms}$ & - & $500 \mathrm{~ms}$ & $500 \mathrm{~ms}$ \\
0 & $0 \mathrm{~ms}$ & $0 \mathrm{~ms}$ \\
$\quad \begin{array}{l}\text { Start time: } \\
\text { Gain: }\end{array}$ & $00 \%$ & $0 \mathrm{~ms}$ & & $100 \%$ & $100 \%$ \\
Zero-phase & $100 \%$ & & & \\
band-pass filter: & & & & & \\
$\quad$ High cut: & $250 \mathrm{~Hz}$ & None & - & None & None \\
Low cut: & $60 \mathrm{~Hz}$ & None & - & None & None \\
\hline
\end{tabular}

a Processed digital seismic data plotted on the Versatec plotter for lines 1, 2, 4 , and 5 are shown in Figures 7, 9, 12, and 14, respectively. 


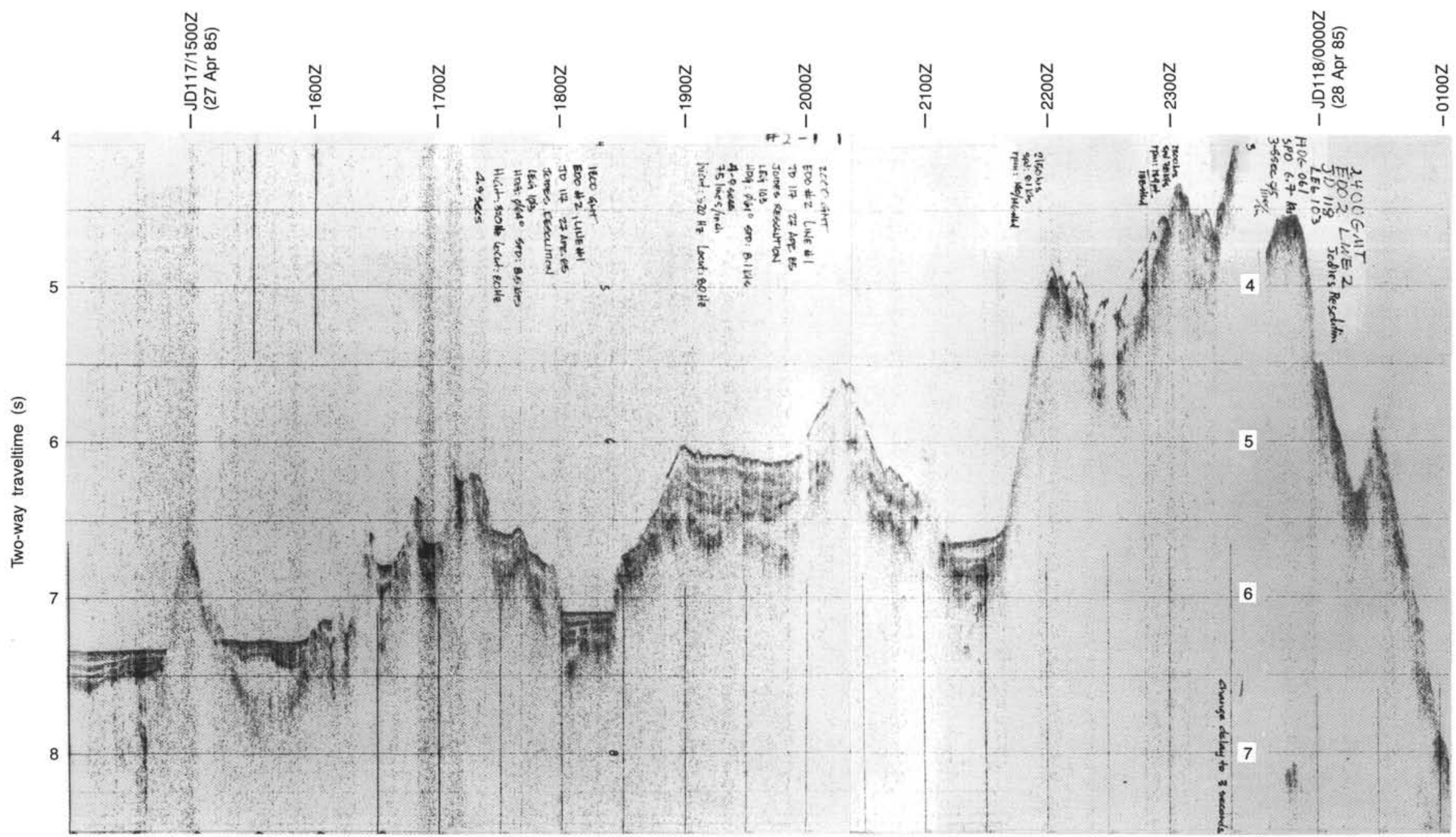

Figure 6. Unprocessed analog seismic data collected on line 1, en route to Site 637 and recorded on the EDO 2 recorder. Trackline navigation is shown in Figure 2. 


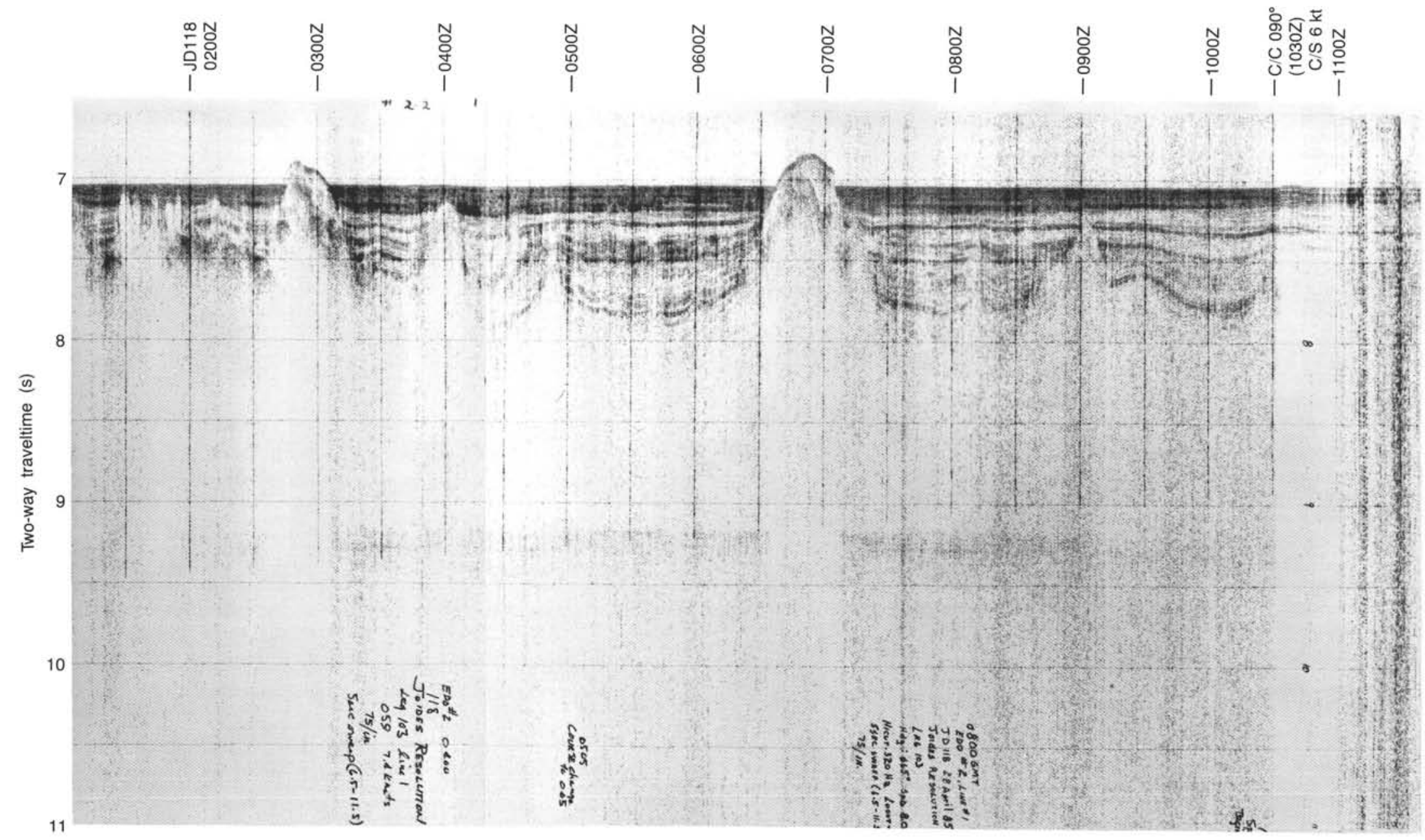

Figure 6 (continued). 


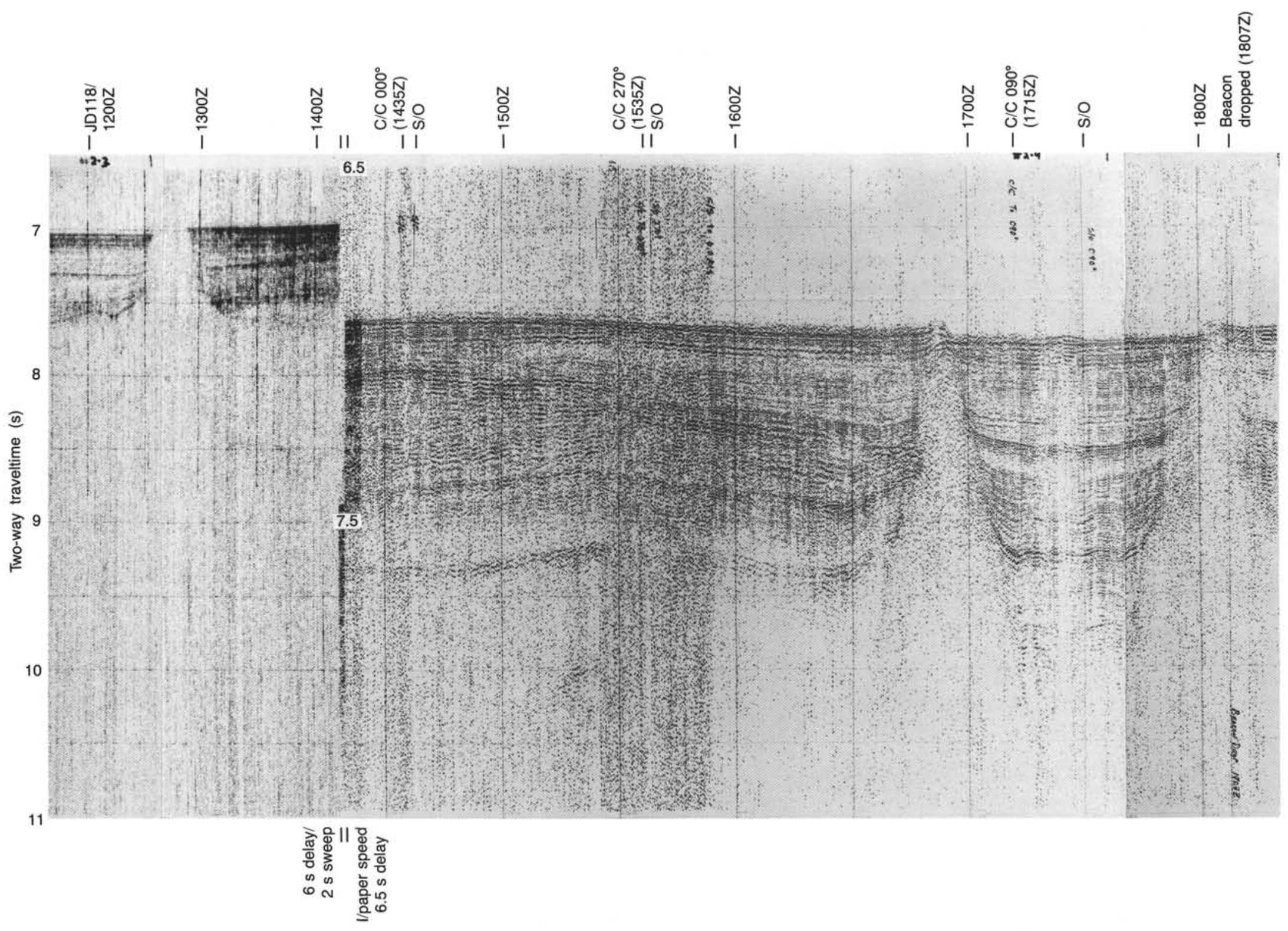

Figure 6 (continued). 


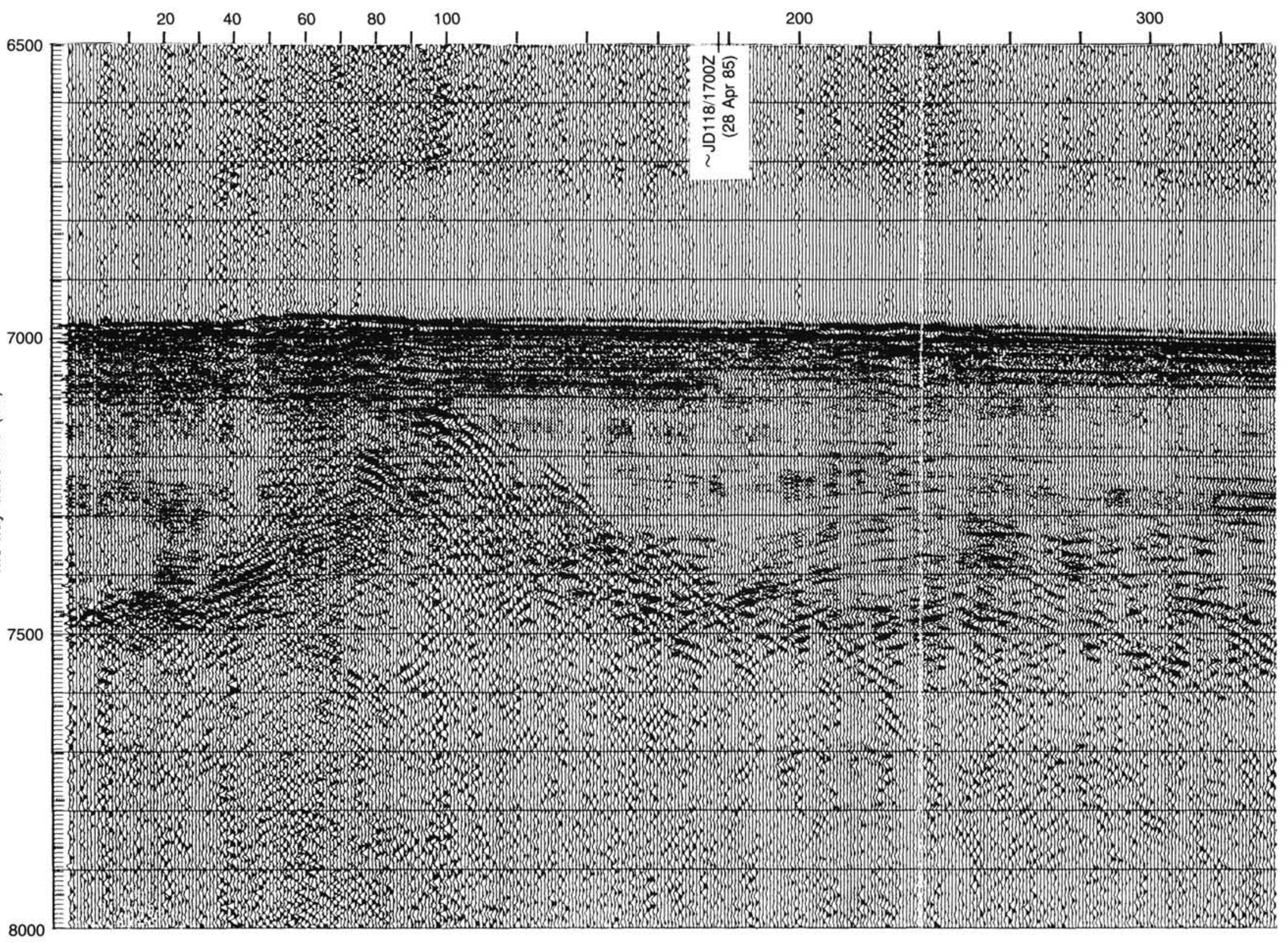




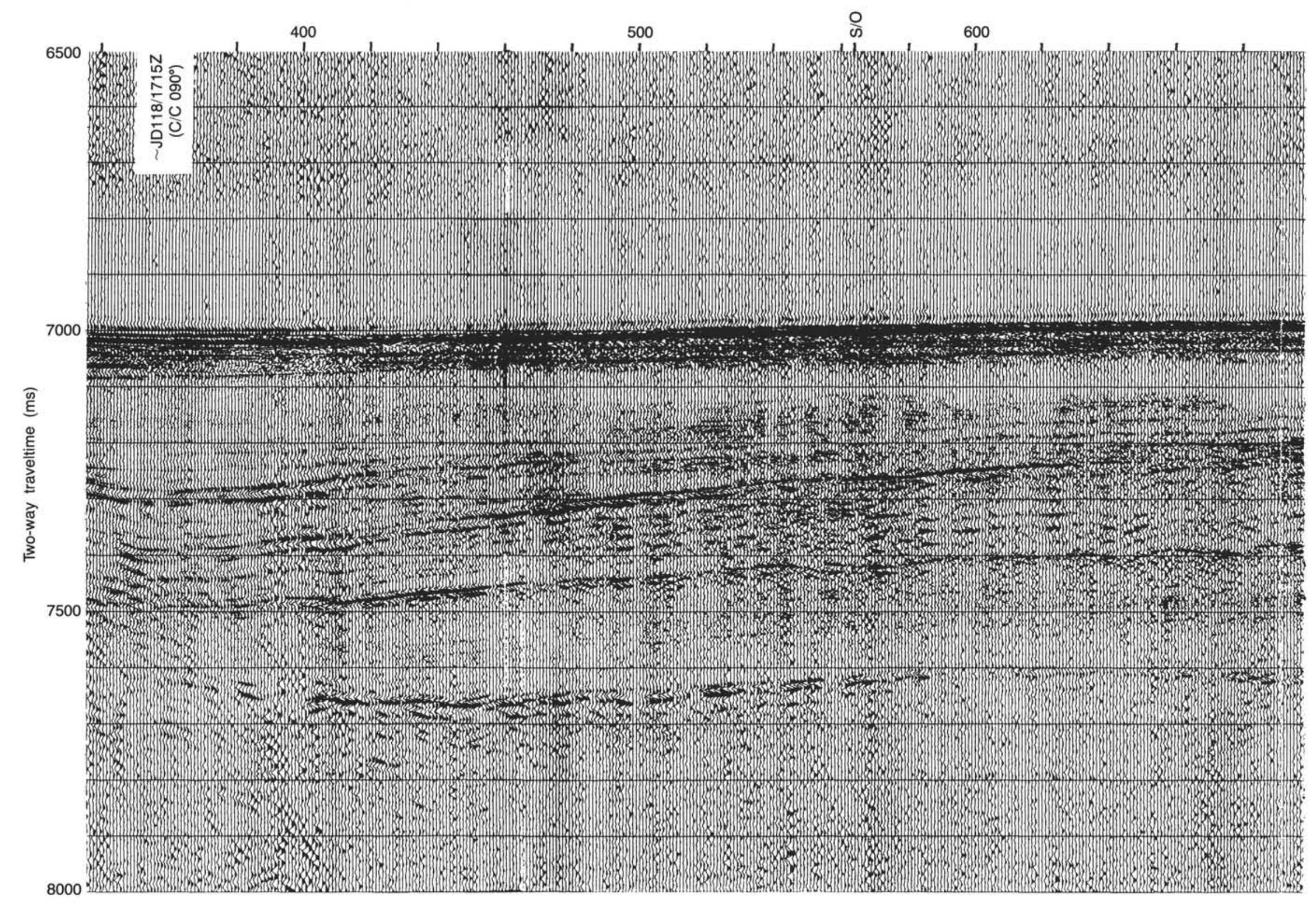

Figure 7. Processed digital seismic data collected on line 1, en route to Site 637. Profile was plotted on the Versatec plotter. Trackline navigation is shown in Figure 2. 


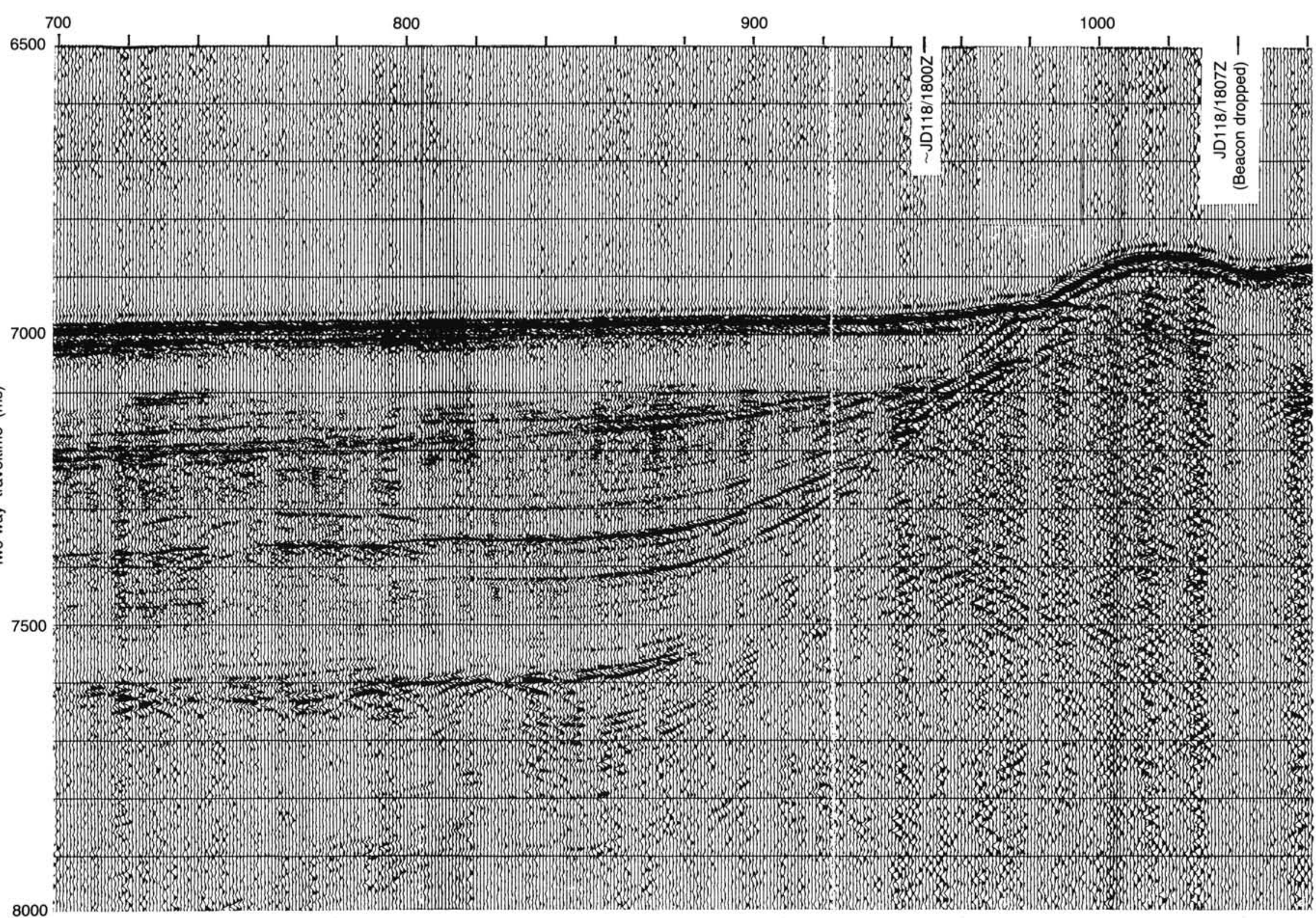

Figure 7 (continued). 


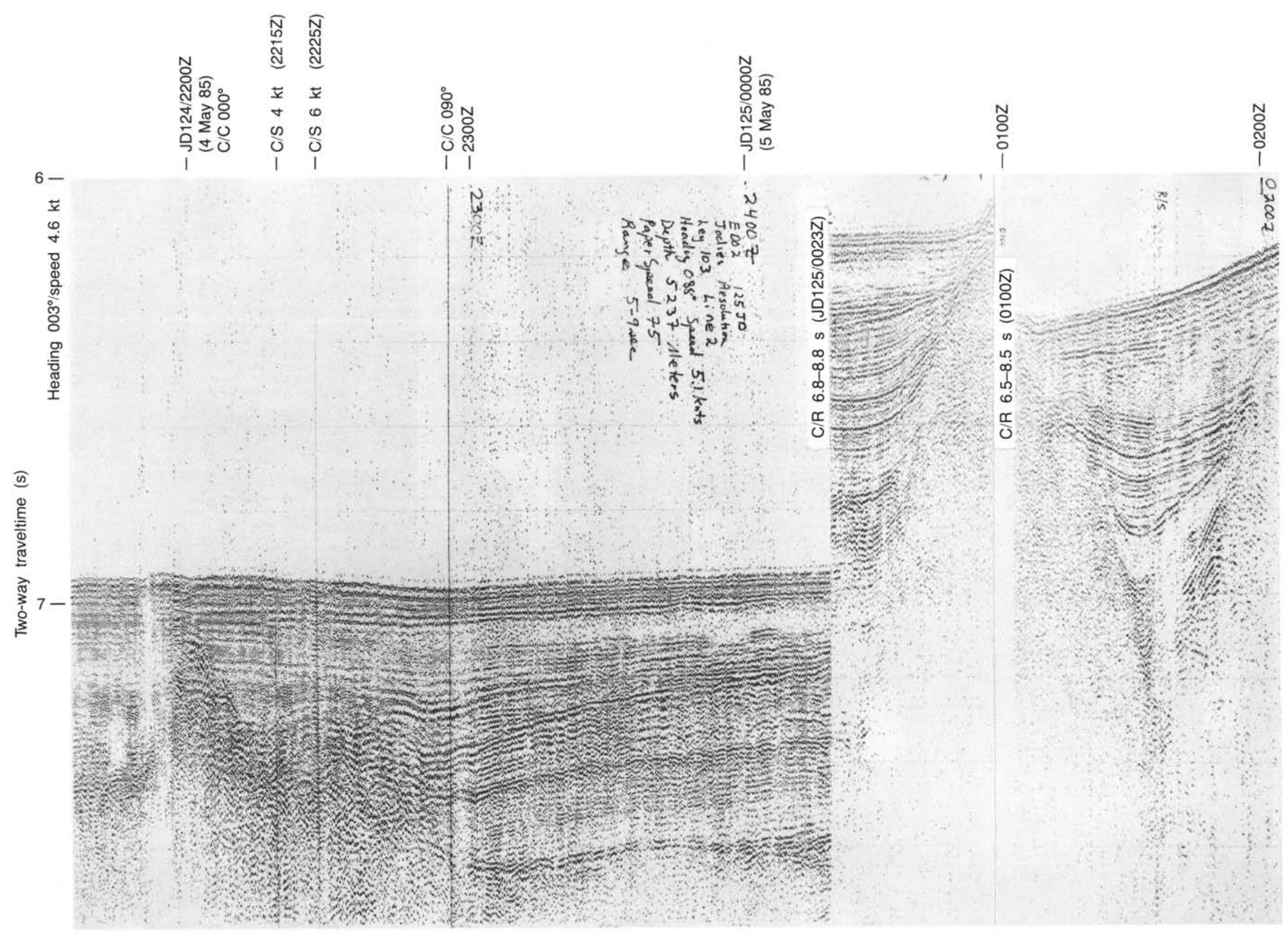




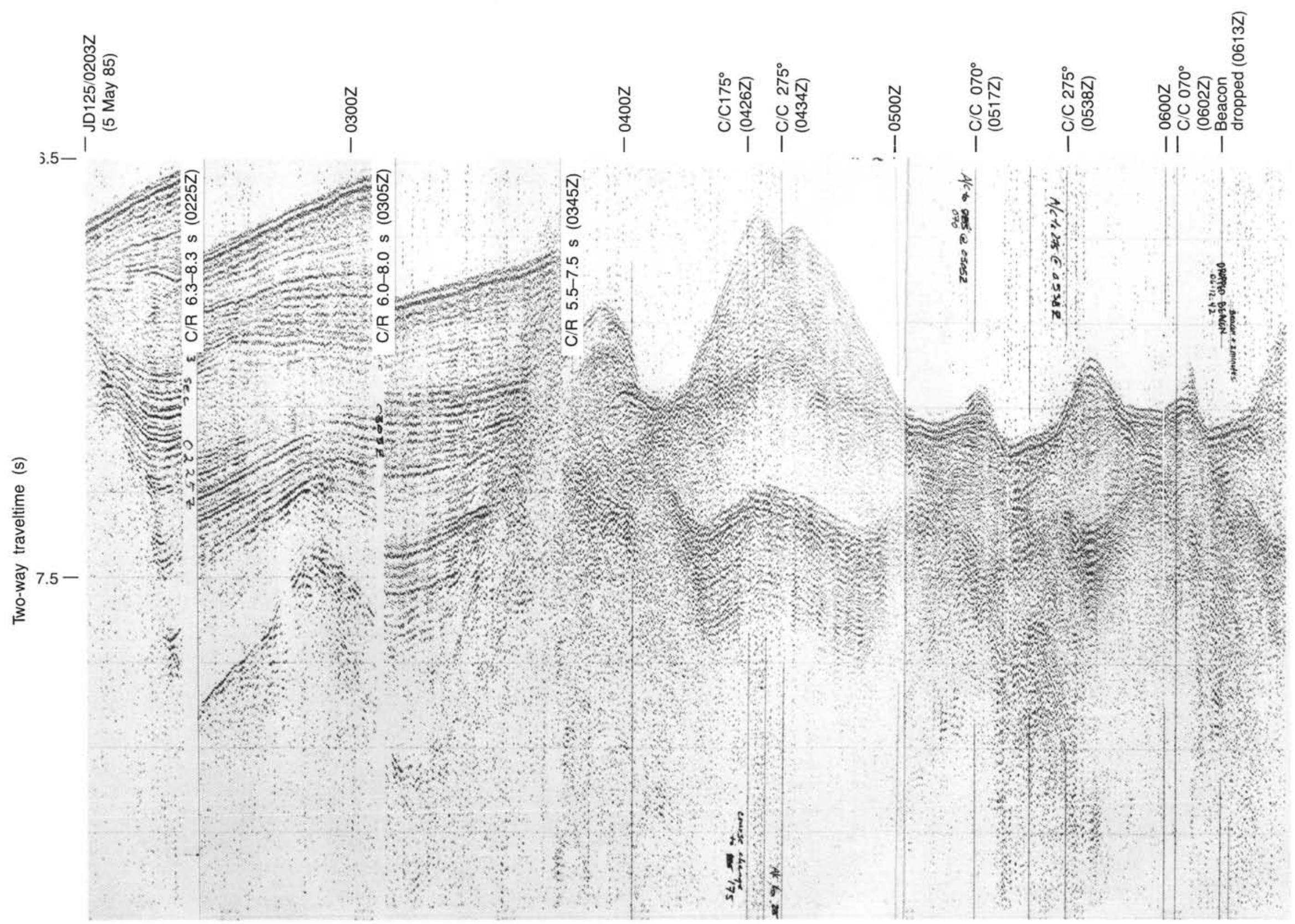




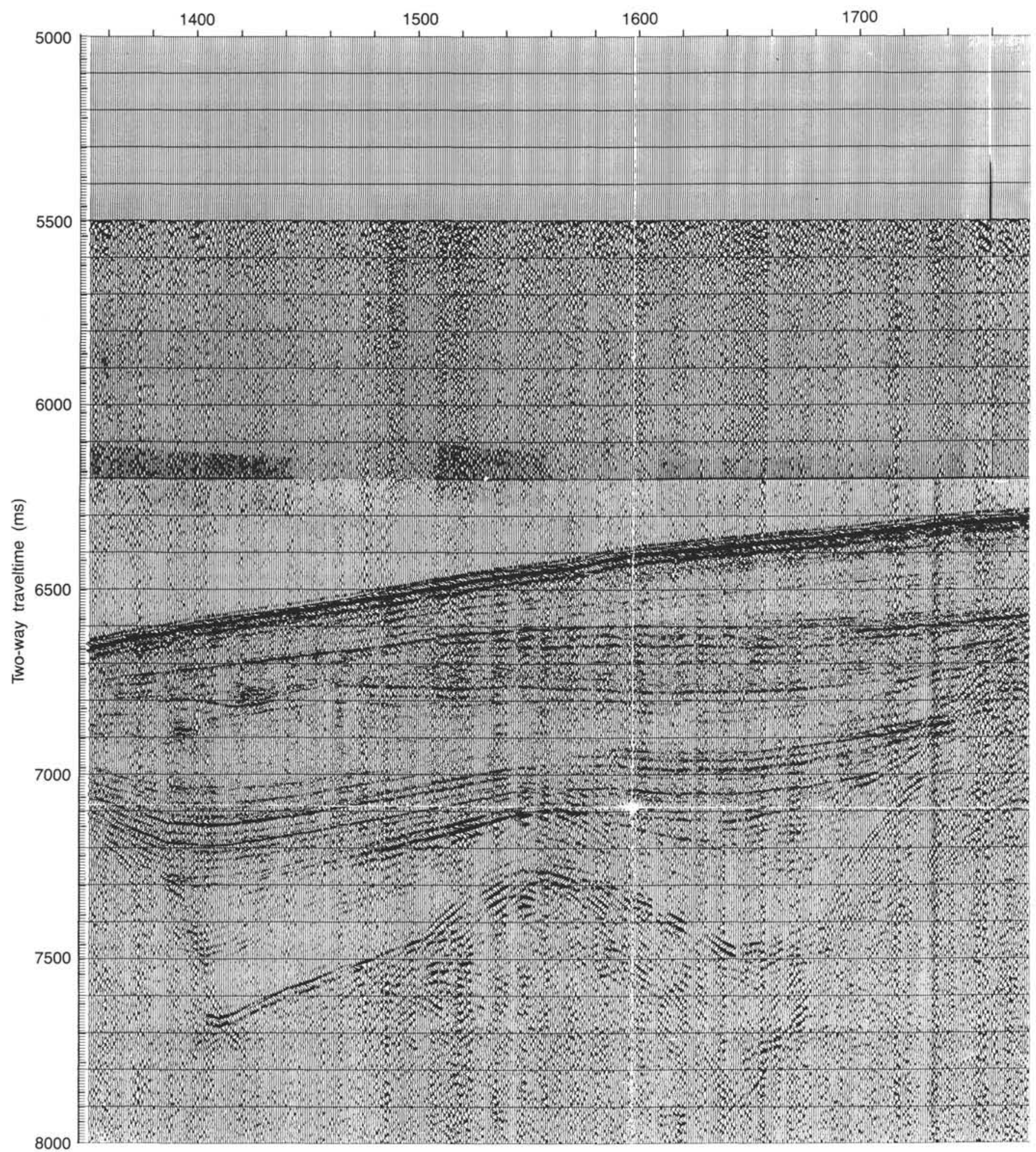

Figure 9. Processed digital seismic data collected on line 2, en route to Site 638. Profile was plotted on the Versatec plotter. Trackline navigation is shown in Figure 3. 


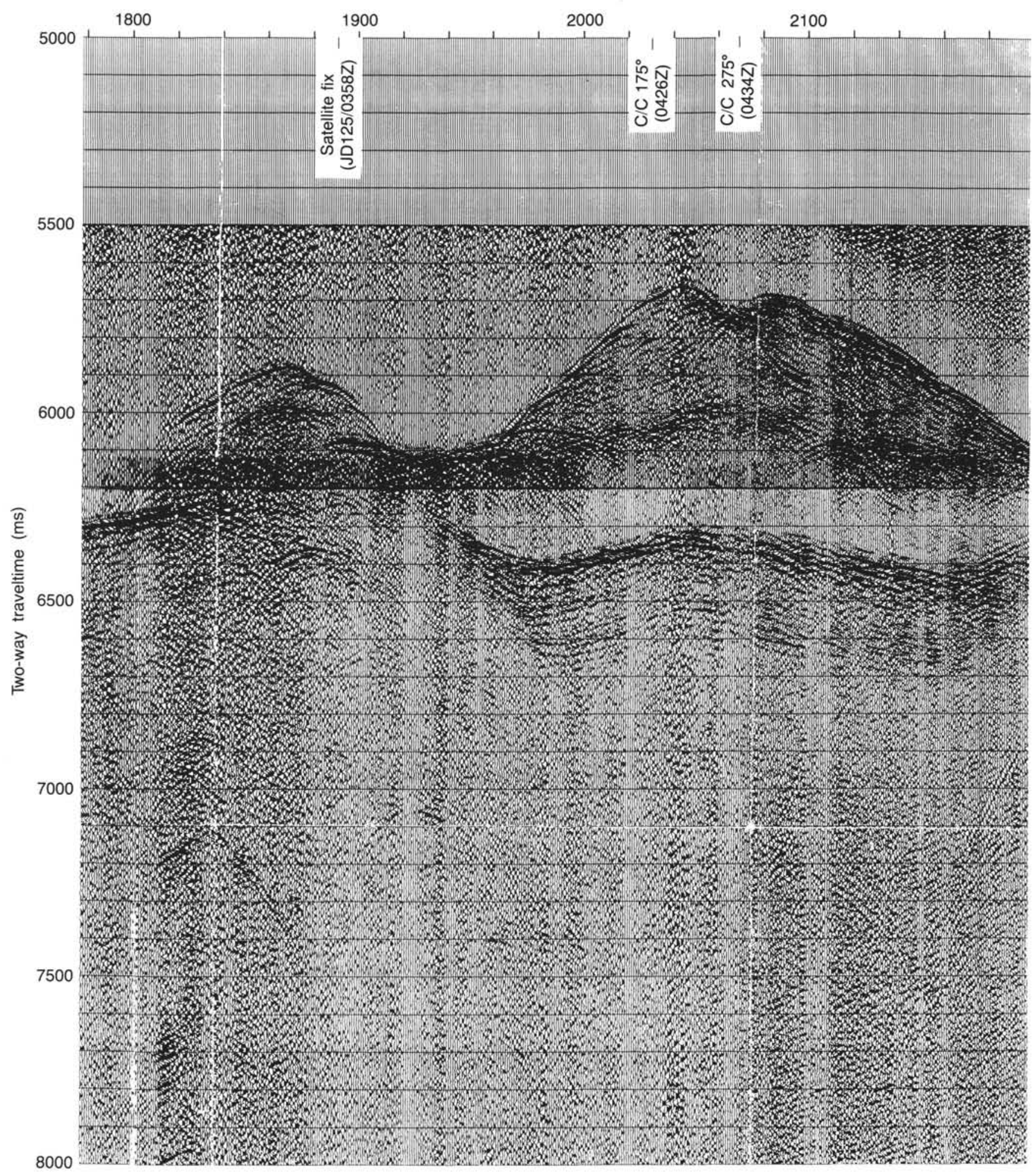

Figure 9 (continued). 


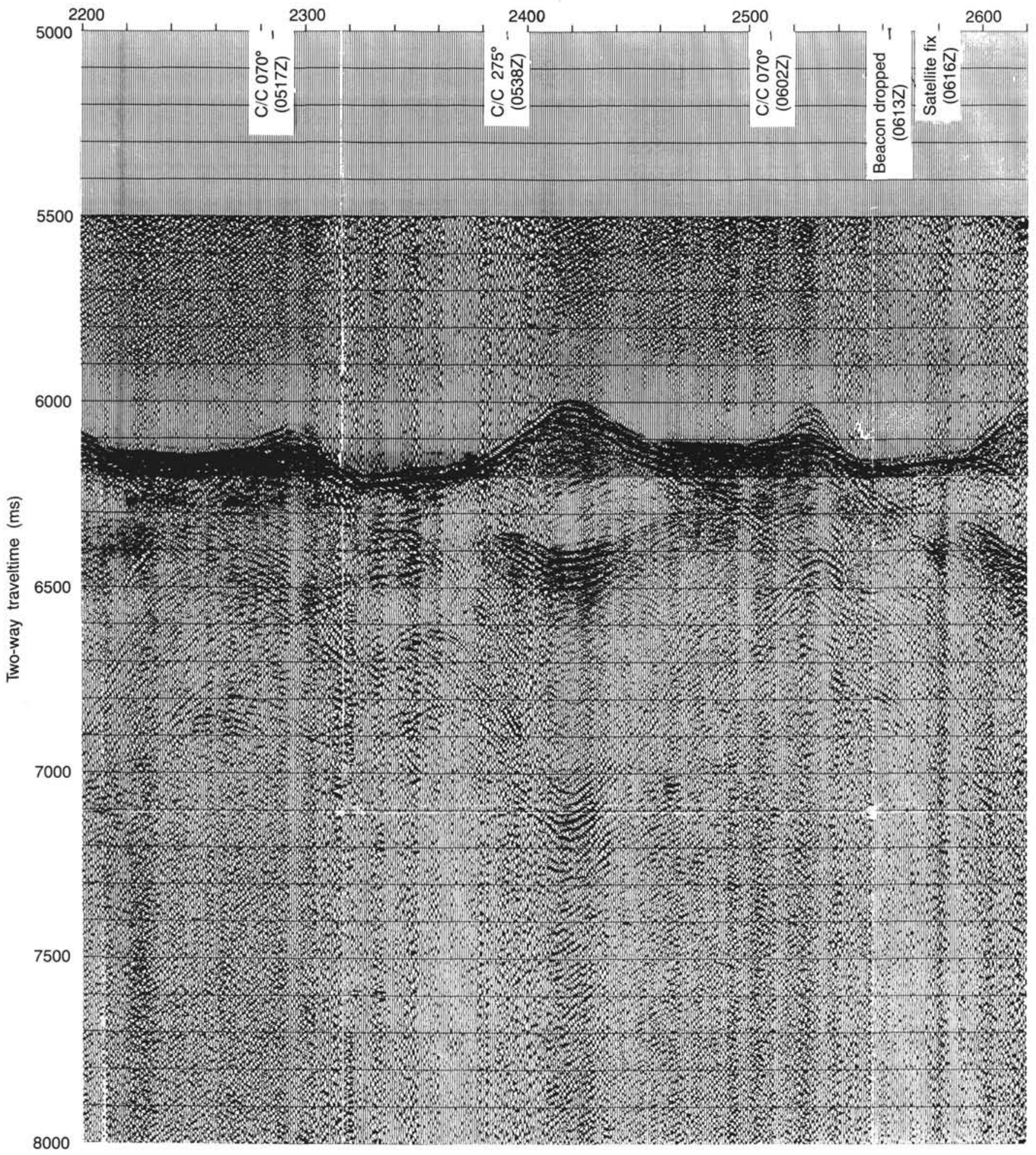

Figure 9 (continued). 


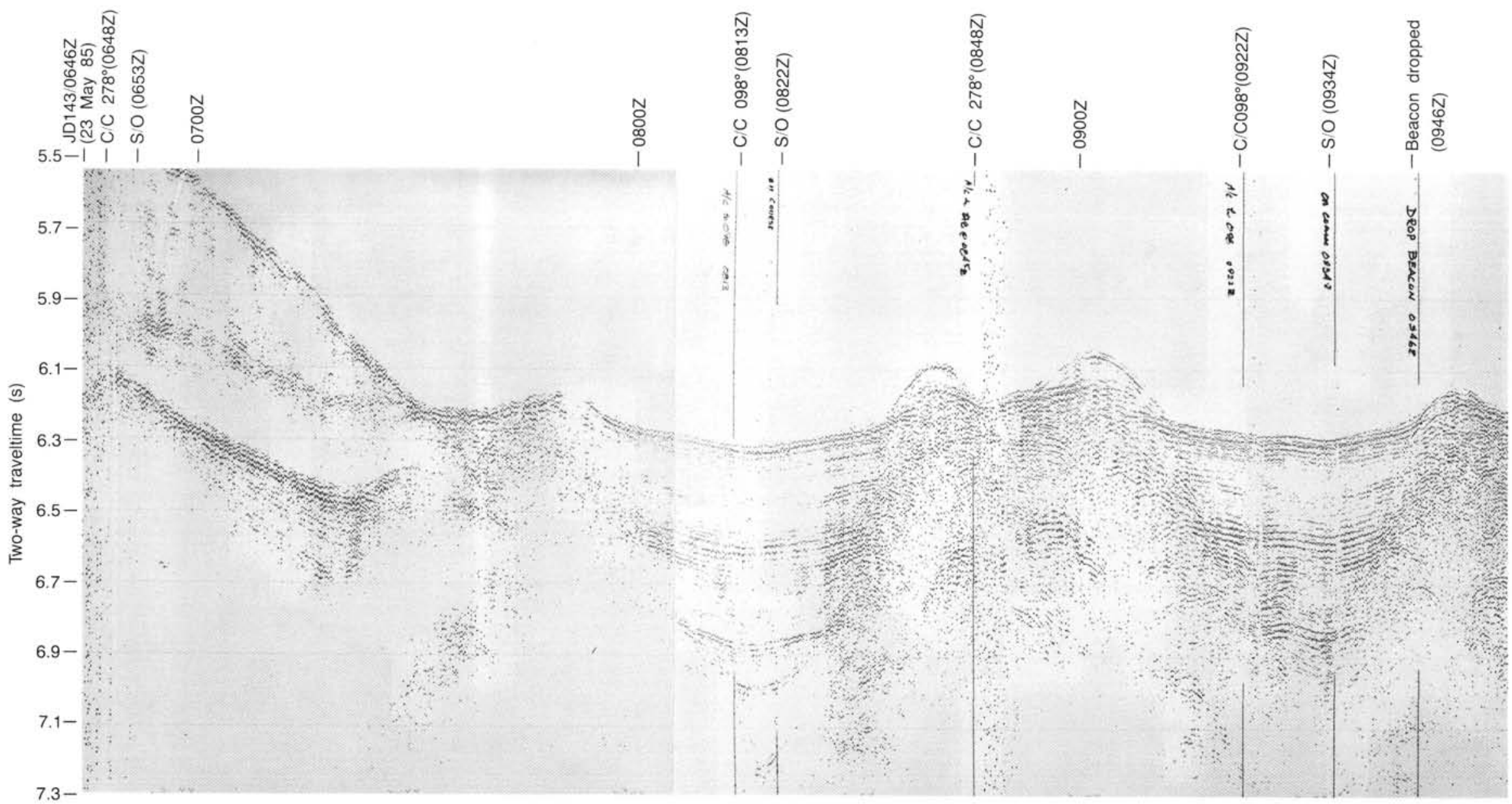

Figure 10. Unprocessed analog seismic data collected on line 3, during transit to Site 639 and recorded on the EDO 2 recorder. Trackline navigation is shown in Figure 4. 


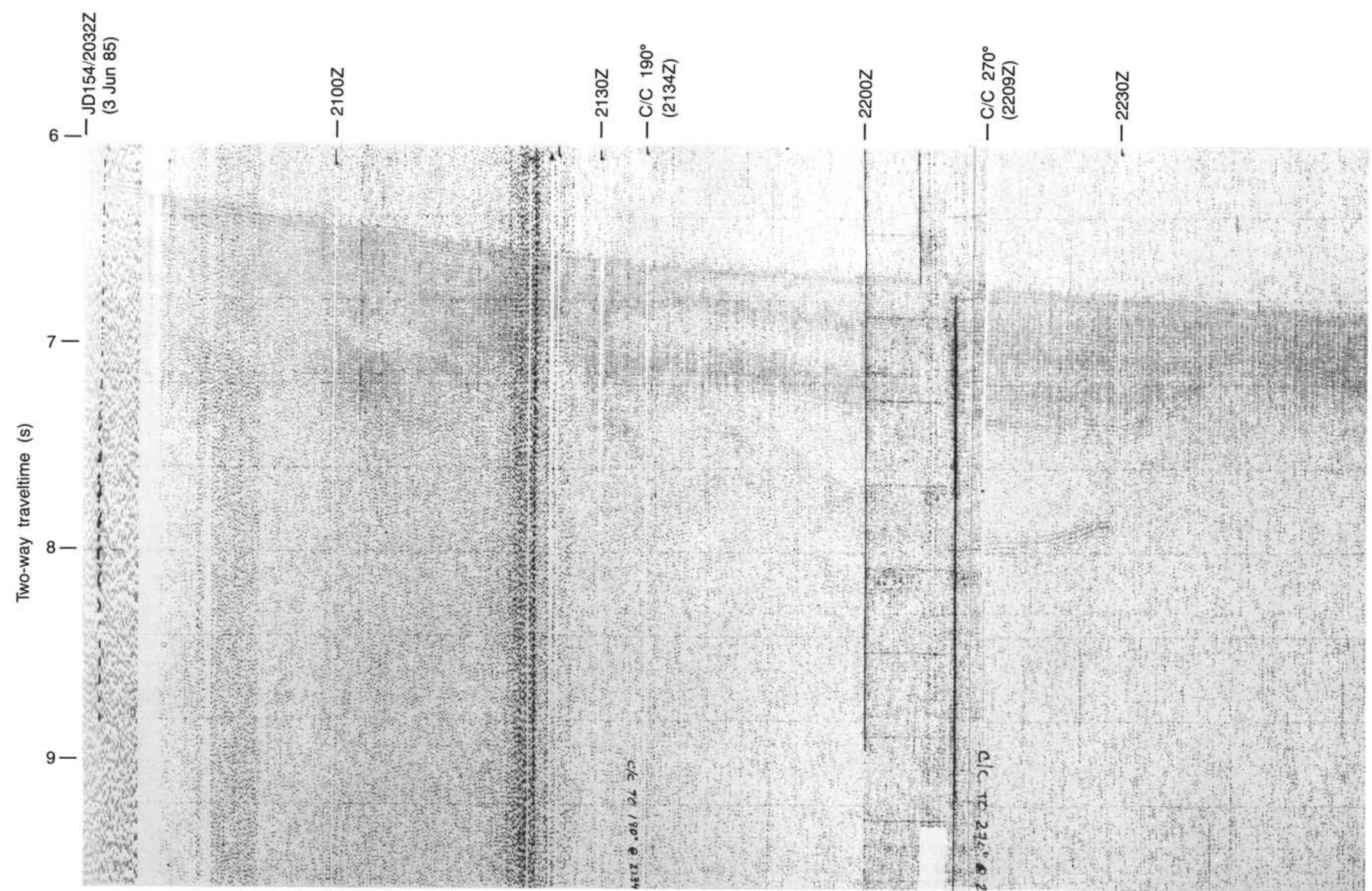

Figure 11. Unprocessed analog seismic data collected on line 4, during transit to Site 640 and recorded on the EDO 2 recorder. Trackline navigation is shown in Figure 5. 


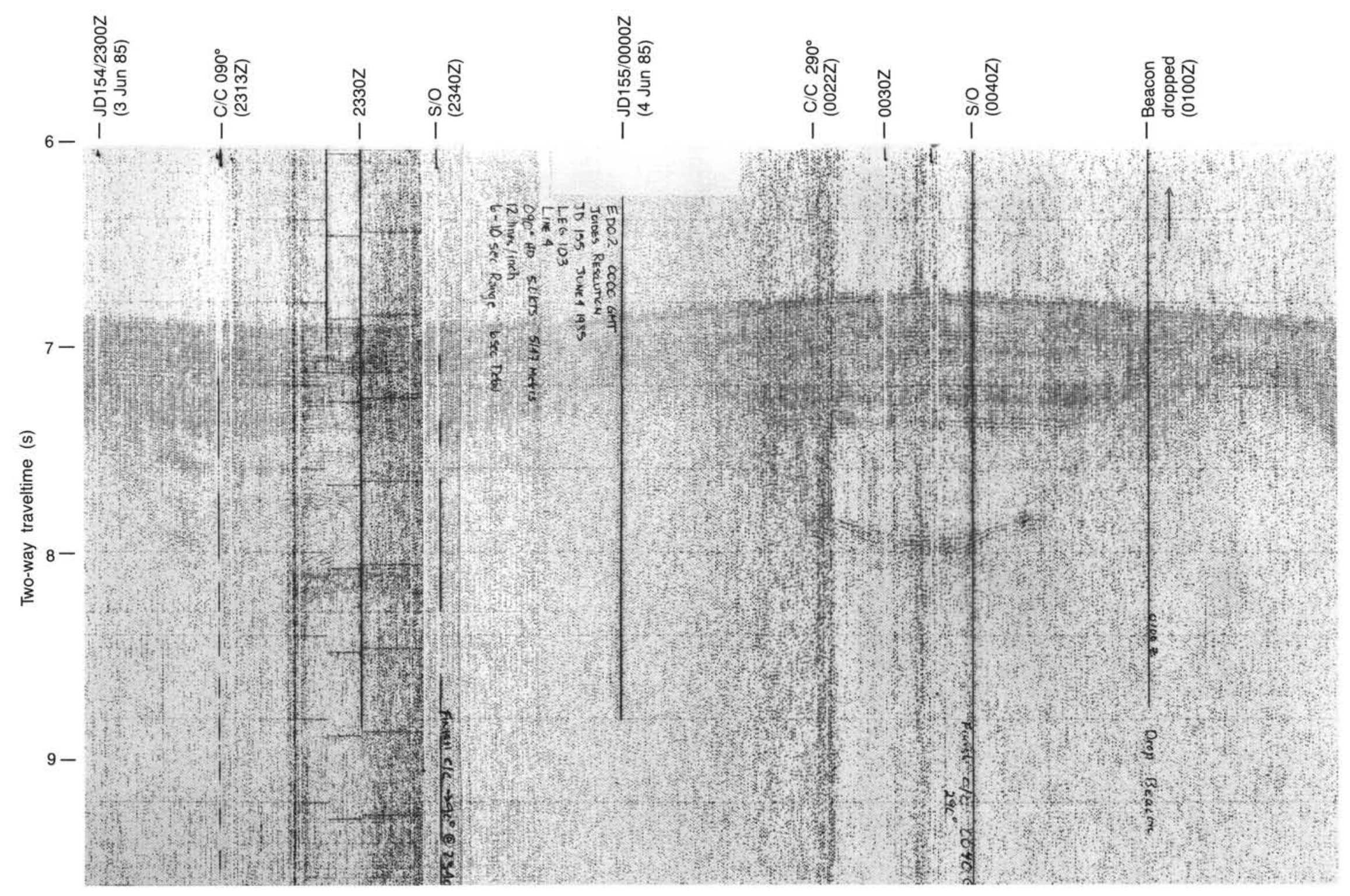

Figure 11 (continued). 


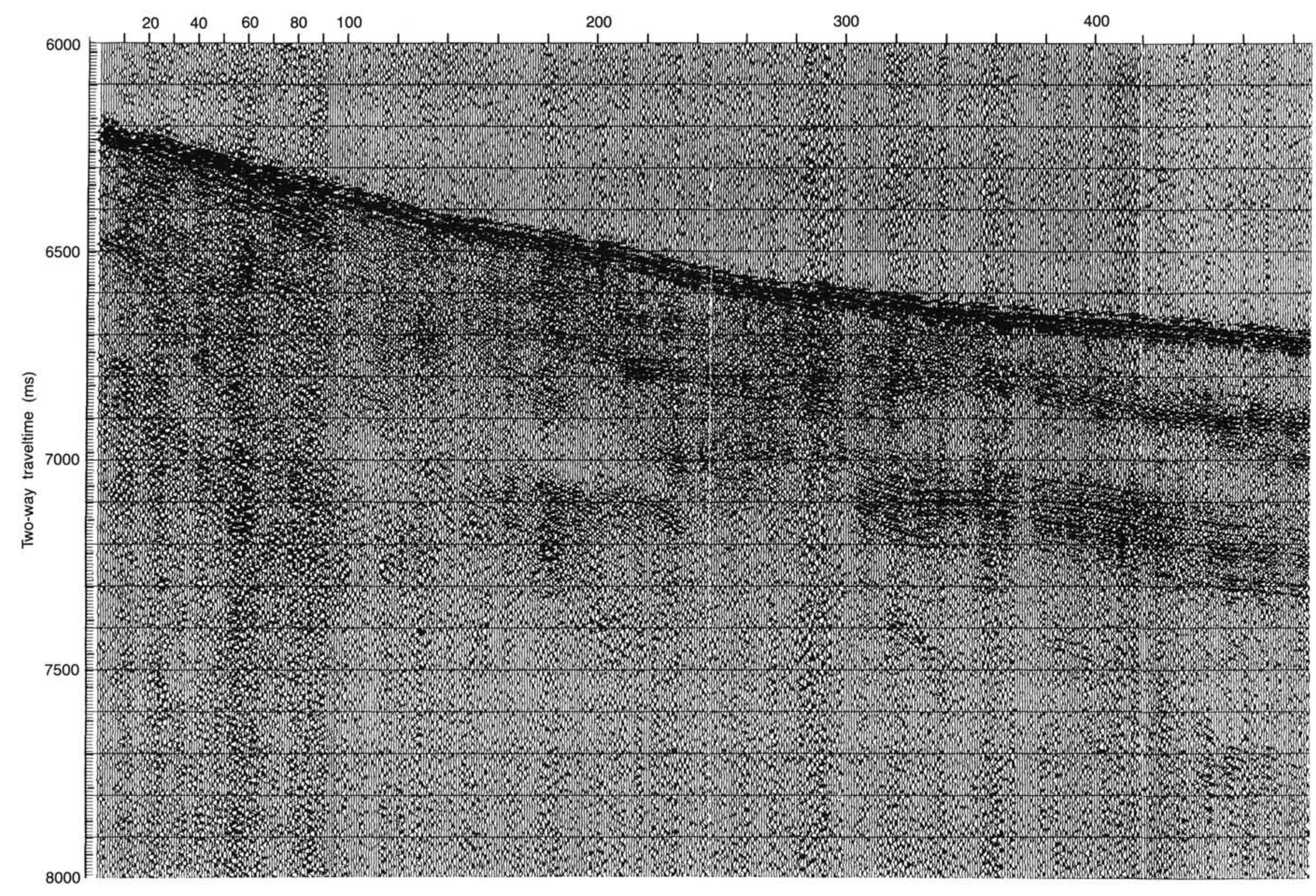

Figure 12. Processed digital seismic data collected on line 4, en route to Site 640. Profile was plotted on the Versatec plotter. Trackline navigation is shown in Figure 5. 
6500

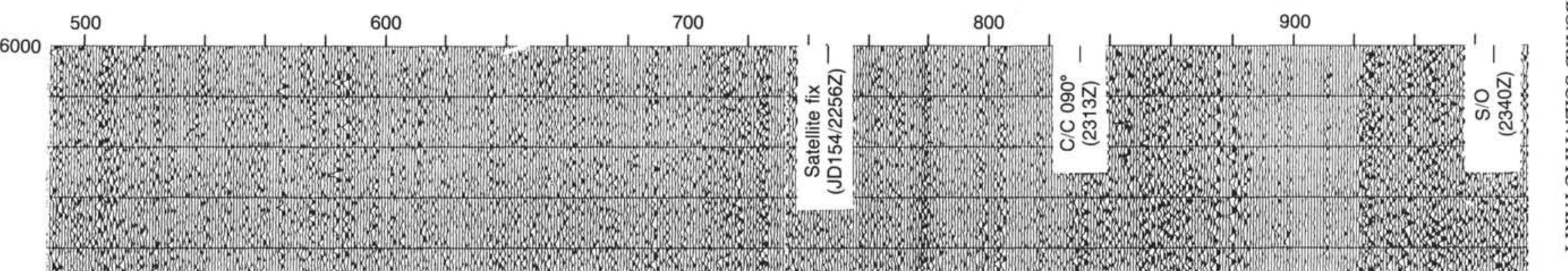

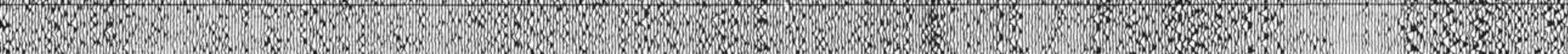

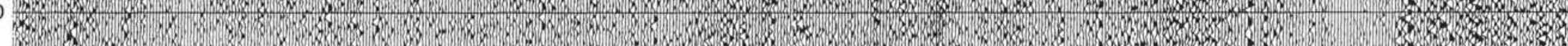

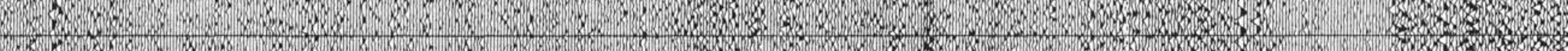

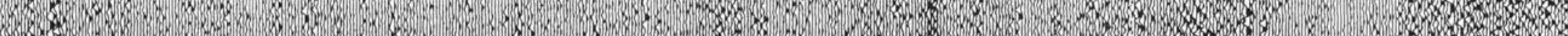

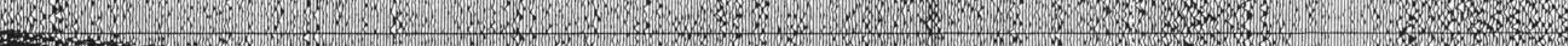
๑

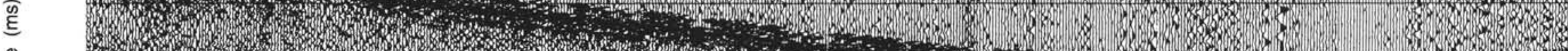

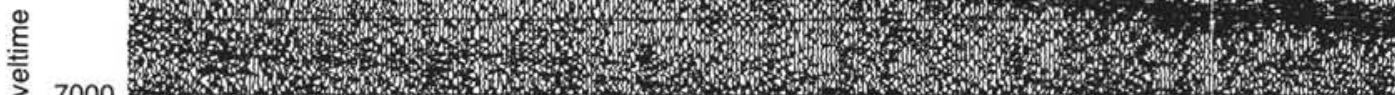
7000 1. 1.7. 1.

1.7.

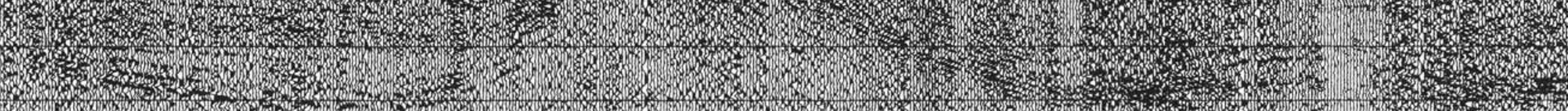
4.

7500

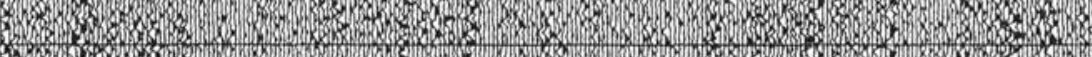

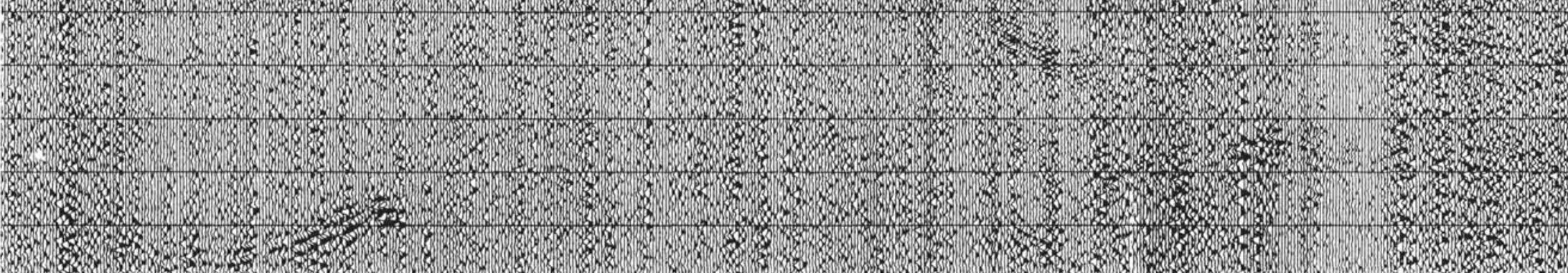
${ }_{8000}$

Figure 12 (continued). 


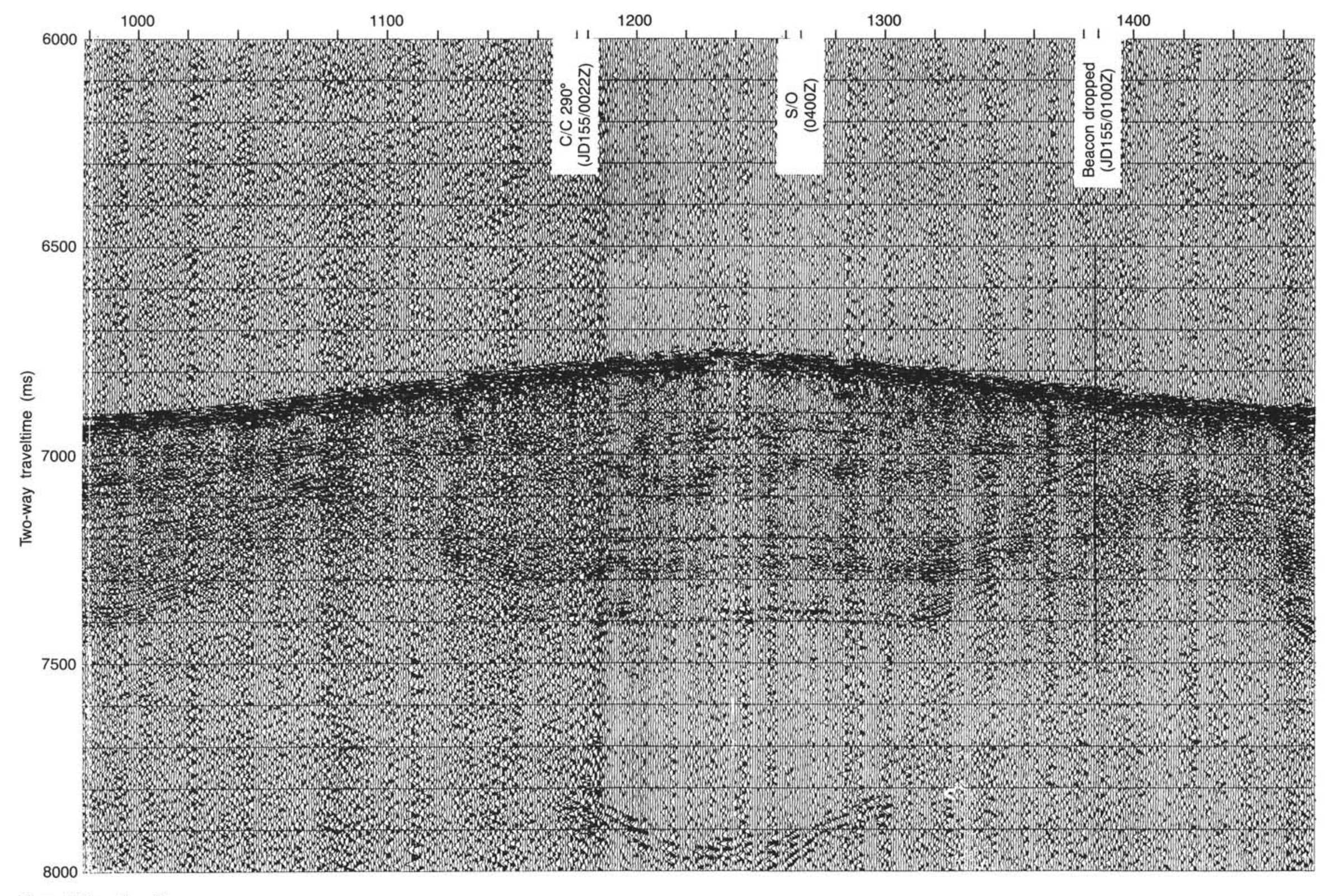

Figure 12 (continued). 


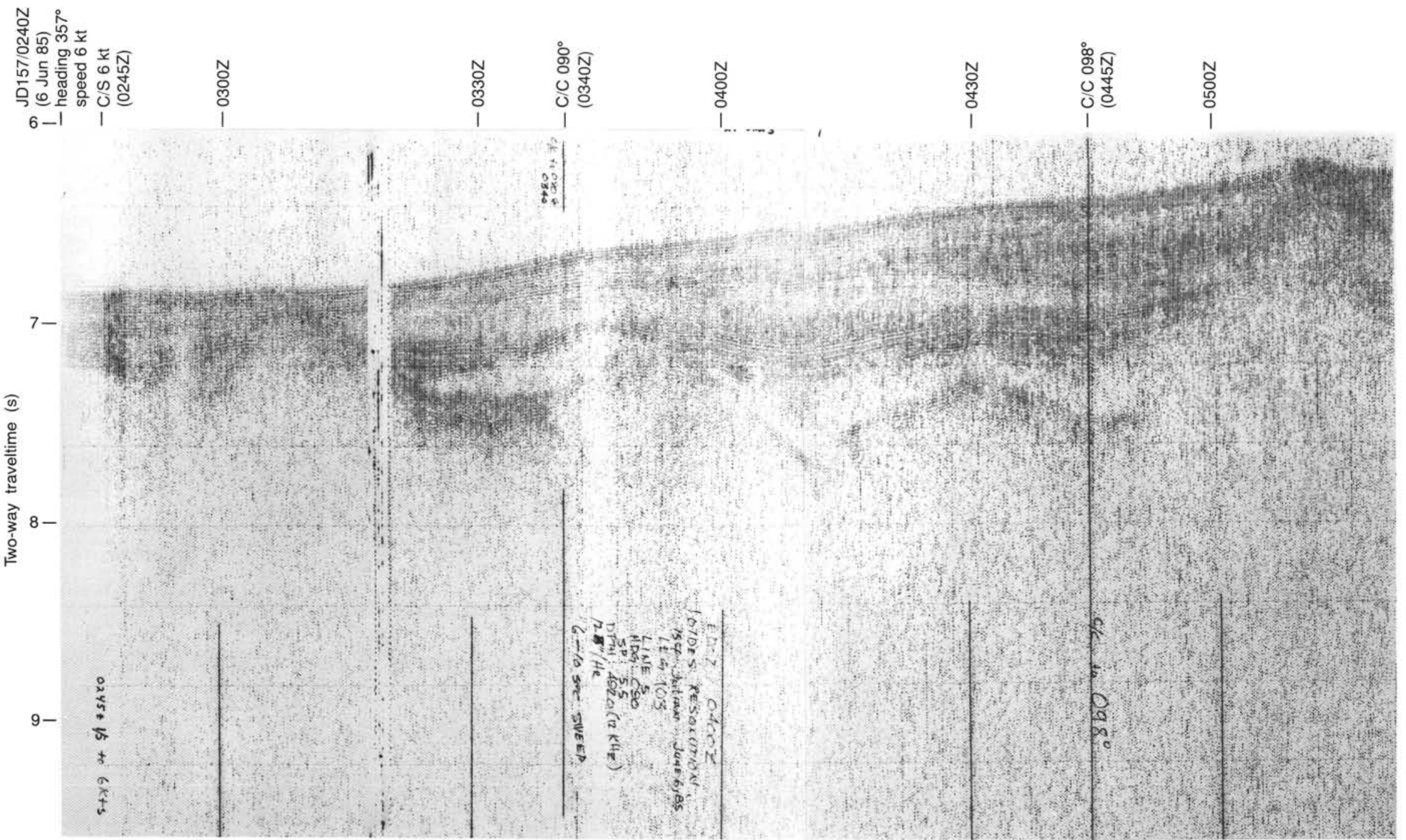

Figure 13. Unprocessed analog seismic data collected on line 5, during transit from Site 640 to Site 639 and recorded on the EDO 2 recorder. Location of profile is shown in Figure 1 B. 


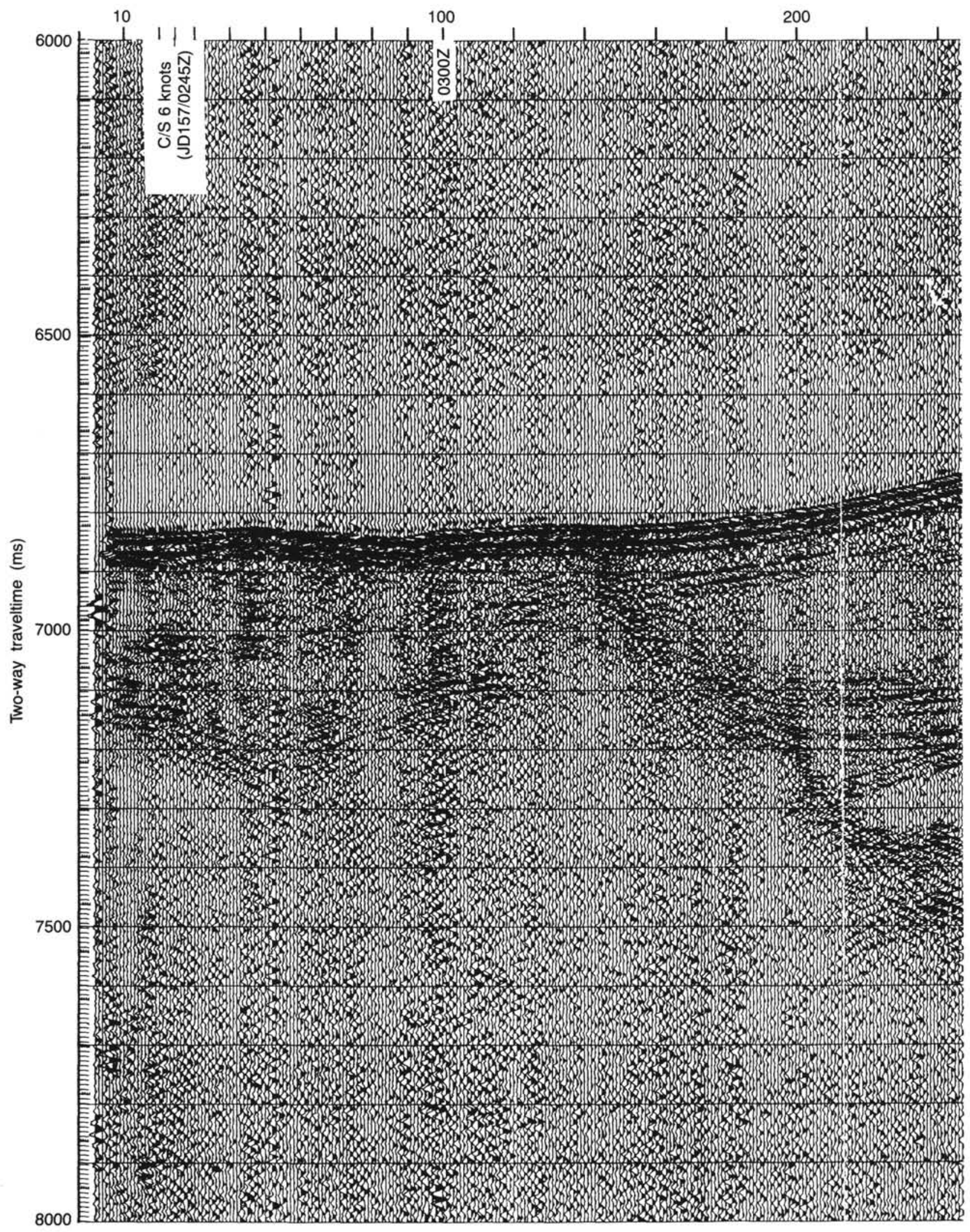

Figure 14. Processed digital seismic data collected on line 5, during transit from Site 640 to Site 639. Profile was plotted on the Versatec plotter. Location of profile is shown in Figure 1B. 


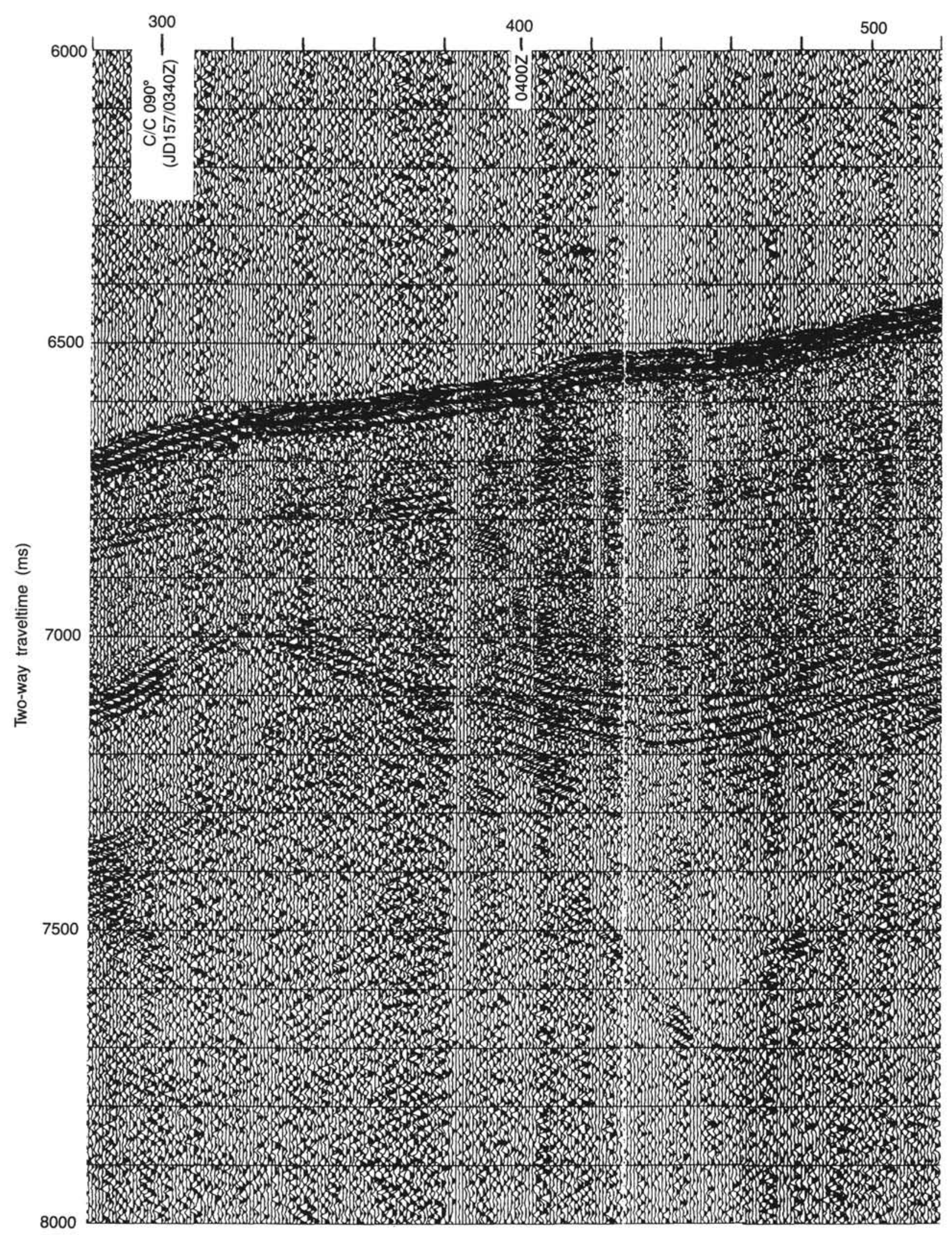

Figure 14 (continued). 


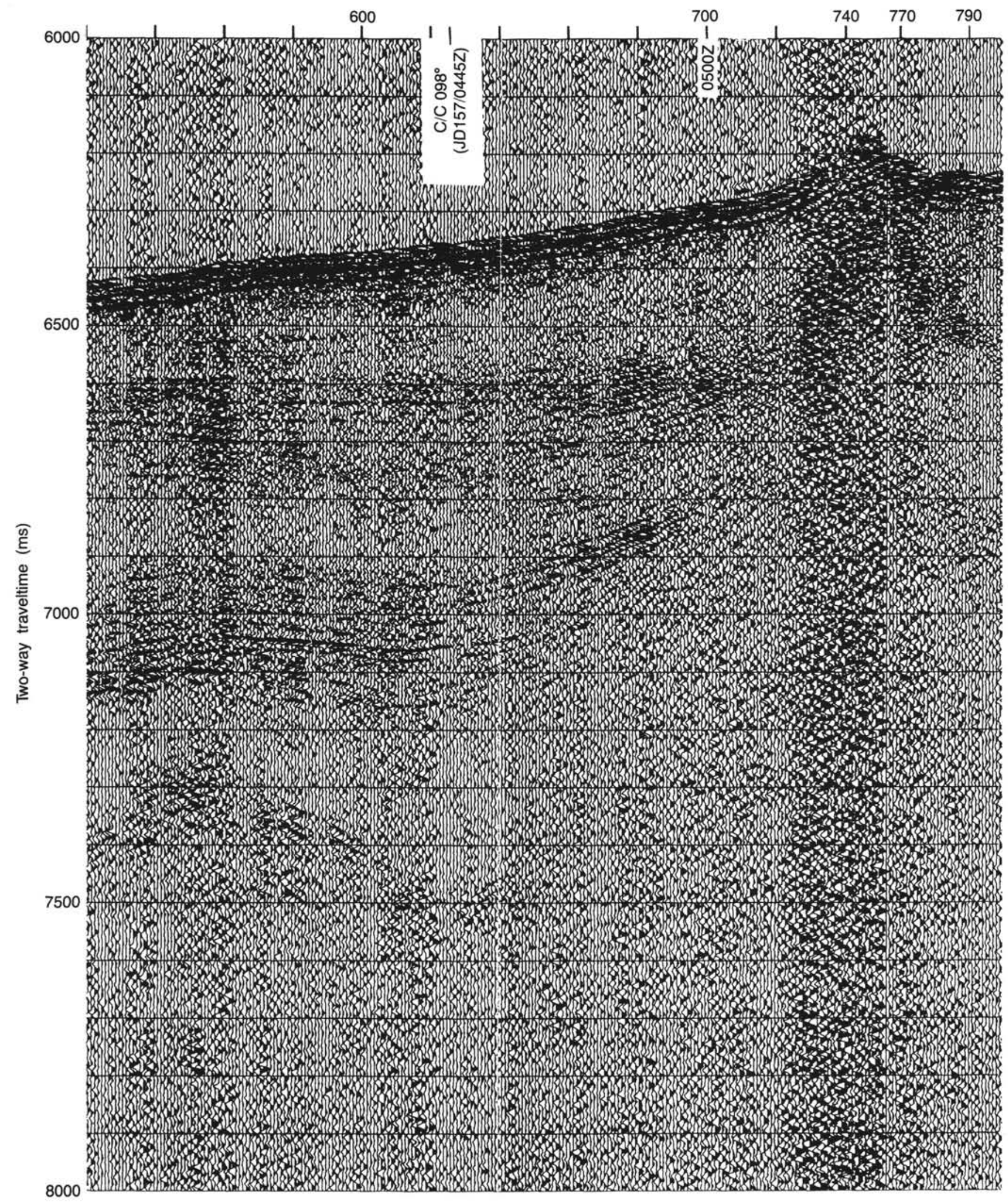

Figure 14 (continued). 